\title{
L'EQUILIBRE BUDGETAIRE EN DROIT POSITIF SENEGALAIS
}

\author{
Par El Hadj Tafsir DIOUF ${ }^{1}$
}

L'équilibre a accompagné l'évolution des mouvements humains en tant que vecteur de stabilité $^{2}$. C'est pourquoi, les gouvernants ont toujours développé des stratégies de quête de l'équilibre qui est devenu un mythe mobilisateur autour du pouvoir politique pour asseoir ou conforter sa cohésion et sa stabilité. Cet équilibre trouve naturellement son prolongement dans les finances publiques. Alors, ses vertus s'amplifient dès lors que s'affirment les enjeux liés à la question de l'allocation des ressources aux différents agents économiques. Les enjeux liés à l'équilibre justifient son érection en principe autour duquel s'articulent les opérations de nature budgétaire. Ainsi, dans sa fonction législative, le droit formalise le principe de l'équilibre dans le droit " posé » ou en vigueur c'est-à-dire le droit positif et plus précisément celui sénégalais. C'est en ces termes que se pose la relation qui s'établit entre le principe de l'équilibre budgétaire et le droit positif sénégalais.

Un budget en déséquilibre est un phénomène très vieux. En effet, sous l'Ancien Régime, le principe voulait certes que les dépenses commandent les recettes. Cependant les recettes fiscales ne pouvant plus couvrir les besoins croissants de la monarchie, celle-ci doit s'endetter, au point qu'à la fin de l'Ancien Régime les intérêts de la dette représentent la moitié des dépenses de l'Etat. Héritant les dettes de l'Ancien Régime, les acteurs des états généraux de la Révolution prennent des mesures nécessaires pour revenir à l'équilibre. Ce sont là les avant-goûts du principe de l'équilibre du budget formalisé.

Dès la révolution de 1789 , on pouvait pressentir des prémices de l'exigence de l'équilibre par les contribuables. A la lecture de l'article 14 de la déclaration des droits de l'homme et du citoyen dans lequel « tous les citoyens ont le droit de constater par eux même ou par leurs représentants la nécessité de la contribution publique, de la consentir librement, d'en suivre l'emploi, d'en déterminer la quotité, l'assiette, le recouvrement et la durée », le contribuable pouvait montrer sa réticence à payer l'impôt dont la nécessité n'est pas avérée. Ainsi le déficit, le recours à des recettes extraordinaires comme l'emprunt ou la création de monnaie, ne font que retarder l'impôt ou créer un impôt déguisé. De ce fait on ferait supporter aux générations futures des charges qui leur sont antérieures.

Donc l'origine du principe de l'équilibre budgétaire pourrait être recherchée dansles finances publiques classiques. En ces moments là, il a été considéré comme clef de voûte des finances publiques classiques et comme une nécessité absolue, parce qu'étant l'expression d'une gestion de qualité. Les tenants des finances publiques classiques, du fait de leur op-

1 DOCTORANT EN DROIT PUBLIC UGB SAINT LOUIS SENEGAL.

2 BALANDIER (G), Anthropologie politique, Paris, PUF 1967, p 37. 
tion pour la neutralité du budget quant au secteur économique, social ..., ont voulu faire de ce principe une règle sacro-sainte. Il le restera pendant longtemps.

Cependant, il faut faire remarquer que ce principe a été remis en cause dans les finances publiques modernes. En réalité, l'équilibre a été maintenu. Seulement, c'est son caractère arithmétique et typiquement budgétaire qui a été dénigré au profit de l'équilibre économique et financier qui peut s'accommoder d'un déséquilibre budgétaire ${ }^{3}$.

La remise en cause du principe de l'équilibre budgétaire a coïncidé avec des évènements imprévus et malheureux comme la première guerre mondiale et la crise de 1929. On a même pensé à des théories contraires à savoir le principe de déséquilibre budgétaire ou le recours au déficit budgétaire. Celui-ci consiste à augmenter les dépenses publiques, quitte à créer un déséquilibre budgétaire en cas d'insuffisance des dépenses et des investissements privés.

Autrement considéré, le principe de déséquilibre budgétaire constitue un instrument d'action sur l'économie; il permet d'opposer à une conjoncture économique défavorable, un déséquilibre budgétaire en sens inverse destiné à le corriger. A titre d'exemple, en cas de conjoncture inflationniste, il sera recherché un excédent budgétaire. Mais également, on peut recourir au déséquilibre systématique préconisé par John Maynard Keynes ${ }^{4}$.

Si la théorie keynésienne a eu un écho favorable au siècle dernier, il va sans dire que de nos jours le leitmotiv qui jalonne les discours de certains chefs d'Etats est « réduire le déséquilibre » de leur budget. Outre le Sénégal qui a toujours était bon élève dans le cadre de l'UEMOA, on peut citer la France et les Etats-Unis d'Amériques mais aussi beaucoup de pays de l'Afrique de l'Ouest comme le Bénin. C'est ce qu'avait déjà compris l'ancien président français François Mitterrand, pour lutter contre le déficit chronique dans lequel son pays était plongé en disant que: « la France doit, comme tout particulier français, ne dépenser que ce qu'elle a ». Aussi dans le cadre de l'Union Européenne, ou le taux du déficit de chaque pays est fixé à 3\% de son Produit Intérieur Brut (PIB), la France et l'Allemagne étaient les deux pays qui s'écartaient le plus de la norme posée par le traité de Maastricht de 1992 et le pacte de stabilité et de progression de 2005. Ainsi dans ces promesses électorales l'actuel président Sarkozy avait promis de baisser le déficit public, et depuis son élection, on note une réelle intention allant dans ce sens. Plus prés de nous, au sein de l'UEMOA, des efforts tendant à baisser le déficit des Etats sont aussi sentis. Ainsi ne sommes nous pas en droit de nous poser la question de savoir si l'équilibre budgétaire est resté le dogme impératif qu'il était ou un principe en résurgence.

3 Au demeurant, il faut remarquer que ces derniers temps, avec la crise de l'euro, et plus précisément celle grecque, certains économistes ont tendance à penser que, du déséquilibre budgétaire dépend largement le déséquilibre économique et financier de la communauté européenne.

4 Économiste et financier britannique (Cambridge 1883 - 1946). Il prôna l'action du gouvernement pour assurer le plein emploi de la main-d'œuvre grâce à une relance des investissements. Sa doctrine a eu une influence considérable sur la pensée et les politiques économiques du XXe siècle. 
Le droit positif sénégalais n'est pas resté muet par rapport à ce principe du fait de son importance incontestable et de son actualité remarquable ${ }^{5}$.

Mais qu'entend-on par « droit positif »? Nous empruntons les mots du lexique des termes juridiques qui, selon lui, s'entend comme « l'ensemble des règles en vigueur dans un Etat ou une communauté internationale, à un moment donné quelque soit leur source ». C'est le droit « posé » tel qu'il existe réellement. ${ }^{6}$

L' « équilibre » quant à lui est défini de manière générale comme « une juste combinaison d'éléments assurant la stabilité » ${ }^{7}$. Pour autant la définition spécifique qui s'attacherait à la problématique de notre étude appréhende l'équilibre comme « une situation d'un budget dans lequel les dépenses inscrites sont égales aux recettes inscrites ${ }^{8}$. C'est un équilibre budgétaire qu'on retrouve dans le projet de loi de finances, où le Gouvernement établit un équilibre budgétaire prévisionnel qui résulte de la comparaison faite entre recettes et dépenses inscrites dans la loi de finances et exprimées à la fin de la première partie, à l'article dit d'équilibre. L'équilibre budgétaire tel qu'exprimé dans la loi de finances (LF) est un équilibre formel.

L'équilibre économique s'apprécie dans le rapport sur la situation et les perspectives économiques, sociales et financières de la nation; le Gouvernement présente ses hypothèses économiques pour l'année suivante sur lesquelles est établi le projet de loi en ce qui concerne notamment les recettes fiscales. Ces prévisions économiques pour les quatre années à venir constituent l'équilibre économique.

Enfin le projet de loi de finances comporte les autorisations relatives aux emprunts et à la trésorerie de l'Etat et évalue les ressources et les charges qui concourent à la réalisation de l'équilibre financier et présenté dans un tableau de financement. C'est l'équilibre financier.

Quant au terme « principe » il désigne une règle générale d'ordre conceptuel qui guide une idée ou une action. Le principe éclaire la règle budgétaire.

Par analogie à la distinction classique faite entre l'autonomie financière et l'autonomie budgétaire qui implique la conclusion selon laquelle celle là est plus large que celle-ci et l'implique en même temps; on peut aussi dire par induction que l'équilibre financier est plus large que l'équilibre budgétaire et l'implique également.

$\mathrm{Au}$ demeurant, force est de constater que la valeur juridique du principe de l'équilibre diffère selon les niveaux local, étatique, ou communautaire. D'ailleurs les approches qui en sont faites dans les différents textes qui le consacrent sont distinctes. D'abord au niveau communautaire ce sont les dispositions de la directive 06-2009 du 26 juin 2009 relative aux

5 Les nombreux « plans d'austérité » qui sévissent en Europe ont pour objet de combattre les déficits budgétaires des pays en question (Grèce, Espagne...).

6 Lexique des termes juridiques, $14^{\text {ème }}$ édition, Dalloz, 2003, page 231.

7 Larousse 2008, p.299.

8 TAM Nguen Chanh, Lexique budgétaire et fiscal du Sénégal, CREDILA 1982, p.51. 
lois de finances ${ }^{9}$ qui réglementent le déficit au sein de l'Union. Ensuite au niveau étatique, c'est la loi 2011-15 du 8 juillet 2011 portant loi organique relatif à la loi de finances qui pose les bases de l'équilibre du budget de l'Etat. Enfin au niveau local c'est la loi 2013-10 du 28 décembre 2013 portant Code Général des Collectivités Locales qui pose le principe de l'équilibre des budgets locaux ${ }^{10}$. Mais cette règle posée par la loi sera illuminée par un ancien décret 66-510 du 04 juillet 1966 portant régime financier des collectivités locales ${ }^{11}$. Si le principe de l'équilibre a une valeur obligatoire dans le cadre des collectivités locales, il a une valeur politique et psychologique au niveau étatique et n'est qu'un simple indicateur de convergence économique et financière dans le cadre communautaire.

Par catégorisation des principes qui gouvernent le cycle budgétaire c'est-à-dire de la préparation à l'exécution en passant par l'adoption (vote), nous avons les principes récurrents et plus connus de l'unité, de l'annualité, de la spécialité de l'universalité. Ensuite, il y a les principes plus ésotériques du fait soit de leur nouveauté soit de leur « caducité ». Il s'agit respectivement du principe de sincérité et du principe de l'équilibre. D'abord qualifié de principe classique et dépassé, l'équilibre est de nos jours considéré comme un principe en résurgence, qui est en regain d'intérêt. Il faut dire que l'équilibre entretient des rapports très étroits avec la sincérité budgétaire. En effet, l'équilibre suppose « une évaluation sincère des recettes et des dépenses $»^{12}$. Cette proximité entre le principe de sincérité et principe de l'équilibre est beaucoup plus étanche dans le cadre des finances locales parce que l'équilibre réel requis ici est guidé par la sincérité. Cependant, malgré la proximité et/ou les rapports de complémentarité que le principe de l'équilibre entretient avec d'abord le la sincérité et les autres, ils ne feront pas l'objet d'une attention très particulière dans notre étude. Nous en ferons état qu'accessoirement pour illustration de notre propos.

Le sujet présente un intérêt théorique et pratique. Théorique en ce sens qu'il convient de faire l'inventaire de ce qui reste du principe de l'équilibre budgétaire naguère conçu par la doctrine financière classique comme la panacée ${ }^{13}$ des finances classiques. Nous saurons de ce fait, si les prescriptions keynésiennes l'emportent sur le principe de l'équilibre. Pratique dans la mesure où si le cadre juridique de ce principe est sans ambiguïté de la part du droit positif sénégalais, il faut faire remarquer que les garanties posées se sont montrées inadéquates dans tous les niveaux. Ainsi n'est-il pas fort à craindre que le laxisme qui caractérise le contrôle de l'équilibre ne saisisse, par effet d'entraînement, le contrôle du budget tout court. Alors faut-il se satisfaire de ce constat? Quelle est la pertinence de ce principe au regard des problèmes et des enjeux des finances publiques d'aujourd'hui? Mérite t-il, le

9 Article premier de la 06-2009 du 26 juin 2009 relative aux lois de finances.

10 Article 255 du Code Général des Collectives Locales du Sénégal.

11 Décret 66-510 du 04 juillet 1966 portant régime financier des collectivités locales, JO du 23 juillet 1966, p.891 et s.

12 Article 32 de la Lolf de septembre 2001 en France.

13 Remède universel à toutes maladies. Sens figuré prétendu remède à tous les problèmes. 
principe, d'être révisé en vue d'une meilleure adéquation aux exigences contemporaines? Telles seront les réflexions autour desquelles sera organisé la présente étude.

Pour prendre en charge tous ces contours et ressortir tout l'intérêt que recèle ce sujet nous nous poserons un certain nombre de questions. A savoir si le droit positif sénégalais est-il resté insensible par rapport au principe de l'équilibre budgétaire? A supposer une réponse négative, quels sont précisément les textes qui régissent ce principe? Quelle est la réglementation qui en est faite? A quels budgets ce principe s'applique-t-il? Comment le droit positif sénégalais encadre- t-il le principe d l'équilibre budgétaire? En somme quelle est la valeur juridique du principe de l'équilibre en droit positif sénégalais?

Composé de toutes les normes en vigueur dans le pays, le droit positif sénégalais a, dans un premier temps servi, à travers ses nombreux textes, de cadre d'existence juridique au principe de l'équilibre budgétaire. En effet il est énoncé dans les textes tant communautaires, étatiques que locaux. Et dans un second temps, il s'agit de ce même droit positif qui a prévu des modes de contrôle pour protéger ce cadre juridique du principe de l'équilibre. Ainsi même si un système de contrôle s'effectue sur pièce c'est-à-dire sur la matérialité que constituent les différents documents budgétaires, il s'avère que son efficacité est remise en cause par certains doctrinaires qui vont jusqu'à l'assimilé de cérémonial. C'est donc l'inefficacité de ce contrôle qui commande une proposition osée et téméraire sur l'équilibre des budgets des collectivités locales.

Après avoir revisité les textes qui ont énoncé le principe de l'équilibre budgétaire communautaire à étatique en passant par ceux locaux sans oublier ceux des établissements publics, nous verrons les modes de contrôle possibles tout en les soumettant à une appréciation.

Nous envisagerons dans cette perspective en une première partie le cadre juridique $\mathbf{d u}$ principe de l'équilibre budgétaire, avant de voir dans une seconde partie les garanties prévues pour sauvegarder ce même cadre juridique.

\section{$I^{\circ}$ L'équilibre budgétaire au Sénégal, un cadre juridique ambivalent.}

Comme tous les autres principes en droit budgétaire, celui de l'équilibre budgétaire est aussi prévu dans le cadre juridique sénégalais. Il est donc présent dans le droit positif. Cependant, les énoncés de l'équilibre sont différents selon les niveaux étatique ou infra étatique donc ambivalent.

A - Un équilibre instable dans les finances de l'Etat.

L'équilibre est prévu tant dans les textes communautaires qui lient le Sénégal par la ratification des traités internationaux que dans les textes étatiques entrant dans le bloc de légalité et dont le respect est exigé. 
Dans les textes communautaires, l'article 98 de la constitution de janvier $2001^{14}$ dispose que « les traités ou accords régulièrement ratifiés ou approuvés ont, dès leur publication une autorité supérieure à celle des lois...». De plus, l'article 6 du traité de l'UEMOA stipule « les actes arrêtés par les organes de l'union pour la réalisation des objectifs du présent traité et conformément aux règles et procédures instituées par celui-ci, sont appliquées dans chaque Etat membre nonobstant toute législation nationale contraire, antérieure ou postérieure ${ }^{15}$. C'est dans cet esprit que la directive 06-2009 du 26 juin 2009 relative aux lois de finances est devenue directement applicable. Elle a été reprise par la loi 2011-15 du 8 juillet 2011 relative aux lois de finances ${ }^{16}$. En effet, elle pose en son article premier que « les lois de finances déterminent la nature, le montant et l'affectation des ressources et de charges compte tenu d'un équilibre économique et financier qu'elles définissent ». Ainsi, s'en eston rendu compte de la difficulté à aspirer à un équilibre budgétaire comme défini par les financiers classiques, c'est-à-dire un budget dans lequel les dépenses prévues sont strictement égales aux recettes prévues.

L'esprit de la communauté est allé dans le sens de la compréhension de l'objectif visé par l'équilibre arithmétique entre recettes et dépenses. Ainsi a-t-on substitué l'équilibre économique et financier à celui budgétaire tout court qui est plus contraignant. Il s'agit en effet, de faire en sorte que les " mécanismes budgétaires » de certains pays ne conduisent pas l'union dans une spirale de conséquences néfastes qui seraient la cause de budgets en déséquilibre. Les Etats désireux de relancer leur économie, en recourant à la thèse de Keynes, doivent aussi chercher à ne pas nuire les autres par l'effet de l'inflation qui seraient dommageable à leur économie respective. Il est recherché une discipline budgétaire au sein de chaque pays de l'union.

De ce fait, le principe de l'équilibre budgétaire est de portée relative dans le budget de l'Etat. Il est un levier ou une technique de politique économique et financière n'emportant aucune obligation à la charge de l'Etat. En fait, ce qui est recherché, c'est l'équilibre économique et financier qui est différent de l'équilibre budgétaire même s'il faut admettre que par celui-ci on peut atteindre celui-là et vice versa ${ }^{17}$.

Cependant, dans la nouvelle directive numéro 06-2009/CM/UEMOA portant lois de finances au sein de l'UEMOA, on note une certaine avancée dans le sens de la clémence d'un budget en déséquilibre, mais en même temps dans le sens de la contrainte vers l'équilibre. En effet, ce texte qui entre en vigueur au courant de l'année 2010 dispose en son article 3 que « les lois de finances déterminent la nature, le montant et l'affectation des ressources et des charges de l'Etat ainsi que l'équilibre budgétaire et financier qui en résulte, compte tenu

14 Loi n²001-03 du 22 janvier 2001 portant constitution, JO n spécial du 22 janvier 2001, p. 27.

15 Traité de l'UEMOA, l'économie ouest africaine, banque centrale des Etats de l'Afrique de l'Ouest, $n^{\circ}$ 440-Août-Septembre 1994.

16 Loi organique relative aux lois de finances 26 juillet 2011.

17 Confère introduction pour différence entre équilibre budgétaire et équilibre économique et financier. 
de la situation et des objectifs macro-économiques des Etats membres et des obligations du Pacte de convergence, de stabilité, de croissance et de solidarité ${ }^{18}$. Une petite analyse de cet article permet de faire ressortir l'évolution voulue par les éditeurs de ladite directive.

D'abord, l'absence de l'expression « équilibre économique » qui lui implique, contrairement à l'équilibre budgétaire et financier, l'intervention d'autres acteurs que l'Etat ${ }^{19}$. Mais ce qui entre en ligne de mire de la compétence exclusive de l'Etat s'avère donc être « l'équilibre financier et budgétaire ». Dans la nouvelle réglementation, la nature, le montant et l'affectation des ressources et des charges de loi de finances ne se déterminent plus compte tenu de l'équilibre économique et financier et même budgétaire, mais compte tenu de la situation et des objectifs macro-économiques, et des obligations du Pacte de convergence, de stabilité, de convergence, de croissance et de solidarité. C'est ainsi qu'on a assisté au changement des termes de référence relatives à l'équilibre dans le cadre de l'union ${ }^{20}$.

Cependant, malgré la directive relative aux lois de finances des Etats membres de l'union, on note une absence de dispositifs de sanctions effectives à l'encontre des Etats dits « mauvais élèves ». Ce qui nous fait dire que ce principe ne pose qu'une référence vers des politiques économiques monétaire convergentes. Il s'agit d'un indicateur de convergence des politiques économiques et monétaires.

Au nombre des objectifs de l'Union, à l'article 4 de son traité de 1994, figure en bonne place la réalisation « de la convergence des performances et «... la convergence des politiques économiques des Etats membres dans le cadre d'un marché ouvert et concurrentiel et d'un environnement juridique rationnalisé et harmonisé» ${ }^{21}$. Cet objectif de convergence a été rendu nécessaire au regard du contexte particulier dans lequel évoluaient les économies des Etats membres de l'Union.

En effet, l'effondrement du cours des matières premières, sources d'importantes recettes d'exportation, dans les années 80 , a constitué une grave crise qui s'est ressentie sur les finances publiques des Etats de l'Union. Dans ce contexte marqué par ailleurs par le poids croissant du service de la dette et une dualité institutionnelle (monnaie unique et politiques budgétaires divergentes) dans la conduite des politiques macro-économiques, les Programmes d'Ajustement Structurels (PAS) menés avec l'appui des institutions de Bretton Woods avaient fini de montrer leurs limites.

Face à cette crise économique profonde, reflétée par une détérioration des principaux indicateurs économiques, la volonté d'approfondir l'intégration économique en complément

18 Directive numéro 06-2009 du 19 juin 2009 relative aux lois de finances abrogeant et remplaçant toutes dispositions antérieures contraires notamment la directive 05-97 du 16 décembre 1997 relative aux lois de finances et ses textes modificatifs. Voir article 87 de la directive 06-2009.

19 Il s'agit des autres acteurs de la vie économie de l'Etat tels que les multinationales, les entreprises, les commerces etc.

20 Voir essaie sur l'équilibre budgétaire au Sénégal, thèse de doctorat d'état, FSJP, UCAD Dakar 2002, p. 15.

21 Traité de l'UEMOA, l'économie ouest africaine, banque centrale des Etats de l'Afrique de l'Ouest, n 440-Août-Septembre 1994. 
de l'Union a été manifeste. Ainsi, la nécessité d'une harmonisation des politiques budgétaires nationales à côté de la politique monétaire commune devenait-elle plus qu'impérieuse. C'est cette optique de mise en cohérence des politiques budgétaires avec la politique monétaire commune qui a favorisé la mise en place d'un dispositif de surveillance multilatérale.

La surveillance multilatérale est un mécanisme communautaire de définition et de contrôle des politiques économiques entre les Etats membres de l'UEMOA. Elle vise à assurer la viabilité du cadre macro économique de l'ensemble de l'Union et le rendre cohérent avec le fonctionnement du marché commun. Cette viabilité du cadre macro-économique doit se réaliser à travers une croissance soutenue du revenu moyen, une répartition équitable des revenus, un solde soutenable de la balance des paiements courants et une amélioration de la compétitivité internationale des économies de l'Union. Il s'agit, en d'autres termes, d'une part, de veiller à une amélioration de la position extérieure des économies de l'Union en vue de les rendre crédibles auprès des partenaires internationaux et d'autre part, de s'assurer que les différentes politiques économiques et sociales définies par les pays membres sont compatibles entre elles et garantissent une convergence durable des différentes économies nationales. Ce sont là les objectifs visés par la surveillance multilatérale.

Si tous les principes applicables au droit budgétaire concourent de manière timide à la réalisation de cet objectif, celui de l'équilibre lui, y participe de manière considérable surtout dans le cadre du contrôle. En effet c'est grâce à lui que les politiques de convergence économique sont les plus harmonisées au sein de l'espace UEMOA. Ainsi, pour les besoins de financement des nécessités de développement et de croissance économique les Etats ont tendance à recourir, de manière spontanée et presque systématique, au déficit budgétaire. Cependant, les effets pervers de cette technique prônée par Keynes peuvent, du fait de la monnaie unique qui est d'usage dans l'espace, peuvent toucher les autres Etats par effet de contagion. C'est le cas de l'inflation.

L'équilibre des budgets des Etats de la communauté économique de l'Afrique de l'ouest est matérialisé par la fixation d'un taux plafond qui serait proportionnel au PIB. Au sein de l'union, il était exigé un taux de 3\% du PIB de chaque pays. Il semblerait que la référence prise de l'union européenne n'est pas, comme dans tant d'autres domaines, adaptée aux nécessités des Etats auxquels il était destiné. En effet, après avoir été presque seul bon élève pendant longtemps, le Sénégal est passé au crible lui aussi pour dépasser le taux de 3\% qui a été fixé. Il s'en est suivi un réajustement de ce taux qui est maintenant de 5\% du PIB de chaque pays. Une justification a priori simple dans la mesure où les Etats africains, contrairement à ceux de l'union européenne, ont besoin de recourir au déficit budgétaire pour essayer de stimuler leur économie et tenter d'atteindre le plein emploi.

Dans les nouvelles directives de l'UEMOA plus précisément celle 06-2009 relative aux lois de finances, il est fait allusion au Pacte de convergence, de stabilité, de croissance, et de solidarité. Ce Pacte est né pour faire face à l'indiscipline budgétaire des Etats membres de l'UEMOA, indiscipline par ailleurs incompatible avec la politique monétaire commune, les articles 63 à 75 du Traité instituant l'UEMOA, ont organisé une surveillance multilaté- 
rale dont le but est d'assurer la cohérence des politiques budgétaires nationales avec la politique monétaire commune.

Cette surveillance repose sur la définition et le contrôle d'un certain nombre de critères de convergence. Mais celle-ci, telle qu'organisée par le Traité, n'a pas donné tous les résultats escomptés en raison de ce que principalement la plupart des critères de convergence n'étaient pas satisfaits par les Etats même si on pouvait relever quelques améliorations.

Ces derniers vont alors se réunir le 8 décembre 1999 pour tirer les leçons de cet échec et prendre un nouvel engagement formel à travers l'adoption de l'Acte additionnel $\mathrm{n}$ ${ }^{\circ}$ 04/99/CM/UEMOA portant Pacte de convergence, de stabilité, de croissance et de solidarité entre Etats membres de l'UEMOA.

De l'analyse des textes communautaires, il en ressort que le principe de l'équilibre budgétaire y est apparu certes mais de manière laconique. Cependant, dans les textes régissant les finances de l'Etat la donne est toute différente. C'est ce que nous allons voir dans la seconde section.

Dans les textes relatifs aux finances étatiques on remarque la prévision d'un équilibre budgétaire certes, mais la réalité s'avère être autre. En effet, l'Etat semble adopter l'équilibre économique et financier prévu par les textes de l'union. On est donc passé d'un équilibre budgétaire virtuel, à un équilibre économique et financier recherché.

Le principe de l'équilibre systématique, d'un équilibre recherché pour lui-même, indépendamment du contexte économique et financier, a presque disparu à l'époque actuelle.

A la lecture de la loi $n^{\circ} 2011-15$ du 08 juillet 2011 relative à la loi de finances, on se rend compte que la référence est faite à la directive 06-2009 du 26 juin 2009 de 1'UEMOA relative aux lois de finances qui prévoit un équilibre économique et financier. Ainsi pour accéder à l'équilibre financier, il est nécessaire de parvenir à un équilibre budgétaire. Si l'on part de l'hypothèse selon laquelle un budget équilibré est celui dans lequel les recettes prévues sont strictement égales aux dépenses prévues, on se rendra compte qu'en fait, cet équilibre budgétaire au sein de l'Etat est virtuel. En effet, un article dit d'équilibre est toujours voté d'ailleurs en priorité sur les articles de la seconde partie du budget. Cet article autorise le Président de la république à recourir à l'emprunt pour couvrir « virtuellement » le déficit qui est noté dans le budget de l'Etat. Il suffit, pour s'en rendre compte de revoir la jurisprudence du Conseil Constitutionnel français dans son arrêt numéro DC 79-111 du 30 décembre $1979^{22}$.

Indicateur de convergence en droit communautaire, le principe de l'équilibre budgétaire est de portée relative dans le budget de l'Etat, il est un levier ou une technique de politique économique et financier n'emportant aucune obligation de résultat à la charge de l'Etat ${ }^{23}$.

22 Revue des Arrêts du Conseil Constitutionnel français numéro DC 79-111 du 30 décembre 1979 sur la loi autorisant le Gouvernement à continuer à percevoir en 1980 les impôts et taxes existants, DC 79-110 du 24 décembre 1979 su r la loi de finances pour 1980.

23 MBODJ (F), Revue « droit sénégalais » $\mathrm{n}^{\circ} 7$ novembre 2008, page 317 et suivants, Presse de 1’Université des Sciences Sociales de Toulouse. 
En réalité cet équilibre n'est qu'invraisemblable car n'assurant pas l'égalité réelle et matérielle des ressources et des charges de l'Etat. Ce genre d'équilibre arithmétique entre recettes et dépenses ne se retrouvait que dans les finances classiques, période pendant laquelle l'Etat était abstentionniste. Là, l'Etat ne gérait que des tâches régaliennes, et les masses financières peu importantes, donc apte à concevoir un budget en parfait équilibre. Ce principe a perdu sa valeur avec les changements de circonstances intervenus après les deux grandes guerres. En effet, les Etats étaient confrontés à des impératifs de reconstruction, et de remise à niveau des économies dévastées. C'est donc la raison pour laquelle la thèse Keynésienne à été expérimentée par les Etats modernes dits interventionnistes. Ils sont appelés ainsi car s'immisçant dans le domaine économique jusque là réservé qu'à l'entreprise privée. Ils sont donc devenus importateurs, exportateurs, commerçants, assureurs, transporteurs, etc. Les premières expériences du déséquilibre budgétaire ont donné des résultats convaincants pour les premiers pays y ayant recouru. C'est ce qui a fait que certains pays comme la France ont eu un déficit chronique durant des décennies suscitant même l'indignation de l'union européenne pour revenir à l'équilibre budgétaire qui concourt à l'équilibre financier de tout espace économique.

En perspective, on peut remarquer que la nouvelle directive de l'UEMOA relative aux lois de finances, accorde certes beaucoup plus de libertés dans l'établissement du budget des Etats membres, mais incite au respect du Pacte de convergence qui est défini « comme un engagement formel souscrit par les Etats membres, en vue de renforcer la surveillance de leurs politiques macro-économiques, en particulier les politiques budgétaires, grâce à la définition de modalités précises d'adoption, de mise en œuvre et d'évaluation des programmes pluriannuels de convergence, de croissance et de solidarité et d'un mécanisme de sanctions. Il vise notamment à renforcer la convergence des économies des Etats membres, à conforter la stabilité macro-économique, à accélérer la croissance économique et enfin, à approfondir la solidarité entre les Etats membres $»^{24}$.

Aussi ne faut-il pas faire remarquer que de l'équilibre financier recèle la garantie d'un équilibre politique et même psychologique au sein des citoyens. Politiquement, cela témoigne de la capacité du Gouvernement en place de s'ériger en régime digne de confiance et dont l'économie n'est pas en stagflation. Plus important, cela témoignerait du respect des conditionnalités juridiques posées par les institutions financière mondiales qui sont entre autres : le respect des droits civiques et politiques, des libertés individuelles et fondamentales, en somme l'existence d'un régime démocratique. Il s'en suit une notoriété et une bonne place dans la politique internationale mais aussi la confiance des institutions financière très importante pour l'accès au financement.

Psychologiquement, c'est la manière de lire le budget consistant à ne dire que les prévisions de dépenses y compris l'emprunt autorisé au Président de la république qui rassure le

24 KAM Olé Alain, « Le pacte e convergence, de solidarité, de croissance, et de stabilité entre les Etats membres de l'UEMOA », mémoire de fin d'étude du cycle supérieur pour l'obtention du diplôme d'Administrateur des Services Financiers. (Burkina Faso). 
commun des sénégalais. Alors que le citoyen pouvant lire une loi de finances se rendra compte de la gravité des conséquences de l'emprunt qui n'est rien d'autre que le transfert des impôts actuels sur les générations futures.

Et voila de l'importance pour ce qui concerne le principe de l'équilibre budgétaire, mais aussi toutes les difficultés qu'en à sa mise en œuvre.

Mais étant concevable que dans les budgets des collectivités locales et même là avec beaucoup de difficultés, les Etats ont changé de ligne de mire en posant comme objectif non l'équilibre budgétaire mais celui économique et financier qui est du reste plus vaste et moins épineux.

Dès l'instant où on s'est rendu compte de la presque impossibilité de concevoir des budgets en parfait équilibre dans les Etats membres de l'UEMOA, on a tourné les objectifs vers la recherche de l'équilibre économique et financier en lieu et place de l'équilibre budgétaire. De nos jours ce qui importe le plus ce n'est pas que le budget de l'Etat soit en excédent, en déficit ou en équilibre, mais que l'Etat soit en équilibre financier et surtout économique. En effet, la loi organique dispose que « les lois de finances définissent un équilibre économique et financier ». Le but du législateur organique était d' «amener le Gouvernement et le Parlement à prendre clairement leurs responsabilités en examinant conjointement les répercussions des mesures qu'ils décident sur la physionomie d'ensemble d'un exercice dont ils avaient d'abord dessiné les principaux aspects ». Ils se réfèrent à un « équilibre économique et financier dont la définition peu varier selon les besoins de la population et de la consommation du pays en écartant la notion rigide et trompeuse d'un équilibre mathématique entre les recettes et les dépenses $»^{25}$.

Ainsi, à la question de savoir s'il existe un rapport de causalité entre équilibre économique et équilibre budgétaire? Une réponse négative mais nuancée s'impose. En effet, on se rend compte que les économies les plus saines et même les plus stables et les plus avancées pourrait-on dire, sont hébergées dans des Etats dont le budget est longtemps resté en déficit.

En ce qui concerne la France qui fait partie des locomotives de l'union européenne en matière économique, elle voit cependant son budget en net déséquilibre dans le courant du mois de juillet 2009. En effet, le déficit devrait passer de 57,6 milliards d'euros à 109 milliards d'euros soit 7,5\% du PIB. Ce déficit ne semble pas inquiéter le Ministère de l'économie et des finances qui prévoit le retour à la normale dans les horizons de 2013. Selon le chef du principal parti d'opposition François Hollande, " ces chiffres sont masqués ». Il parle en effet de plus de 150 milliards d'euro en fin $2010^{26}$.

Quant aux Etats Unis qui constituent la première puissance économique mondiale, le déficit public a atteint des taux record ces dernières années avant de connaître une légère chute avec l'installation d'une nouvelle administration aux politiques économiques diffé-

25 MUZELlEC (R), Finances Publiques, Sirey, $14^{\text {ème }}$ édition, septembre 2006, p. 310.

26 François Hollande estime que ce déficit sera à plus de150 milliards d'euros en fin 2010 dans son ouvrage intitulé « Droit d'inventaire », Seuil, novembre 2009. 
rentes de celles des administrations précédentes. Les explications suivantes sont fournies par les spécialistes. D'abord la diminution des sommes faramineuses dues à la guerre en Iraq et en Afghanistan, et ensuite les promesses électorales de l'administration en place.

Ce qui démontre pour nos deux exemples que le déséquilibre budgétaire d'un Etat n'a pas, en pratique, d'incidences immédiates et directement visibles, dans l'économie du pays concerné, pour ne pas dire inexistantes.

Dans la mesure où l'union économique ouest africaine a réussi à mettre en place une monnaie unique, elle est plus encline à œuvrer pour la réalisation de l'équilibre financier, pendant que l'Etat concerné lui, œuvrera davantage pour réaliser l'équilibre économique. C'est donc la santé financière de l'Etat et de son économie en particulier qui reste le souci majeur des gouvernants.

Ainsi la commission de l'union doit concilier à tout prix, au risque de perdre sa raison d'être, deux exigences contradictoires : l'inévitable recours des Etats membres au déficit pour la relance de leur économie et la nécessaire défense de la monnaie unique contre les risques d'inflation et la stagflation consécutive à l'indiscipline budgétaire de ces mêmes Etats.

Cependant, les Etats membres de l'union doivent éviter les déficits publics excessifs pour ne pas ébranler les économies voisines. D'où l'adoption du Pacte de convergence, de stabilité, de croissance et de solidarité. ${ }^{27}$

Après avoir scruté la manière dont le principe de l'équilibre est conçu au plan supra national et à l'échelle intermédiaire c'est-à-dire étatique, nous en ferons de même pour ledit principe mais cette fois ci à un niveau certes plus bas mais non moins important : le niveau décentralisé. Dans ces finances dites infra étatiques, l'équilibre y est exigé.

B - L'équilibre réel : une obligation légale pour les collectivités locales.

A ce niveau il faut se rendre compte que, contrairement à ce qu'il est convenu de remarquer aux niveaux étatique et supra étatique, le principe tel qu'appliqué aux collectivités locales, est une obligation réelle pour celles-ci. Il n'est cependant pas restreint aux collectivités locales, elle est aussi applicable aux établissements publics.

L'équilibre budgétaire est tributaire du cadre économique qui lui a donné naissance ${ }^{28}$. Pour dire qu'en ce qui concerne les collectivités locales, on note une obligation légale à savoir l'équilibre réel. Sur le plan économique, les recettes avaient pour vocation de couvrir les charges nées des dépenses de nature administrative. En raison de sa simplicité technique, l'équilibre demeurait un indice fort d'une bonne gestion financière. C'est la raison

27 Directive numéro 06-2009 du 19 juin 2009 relative aux lois de finances abrogeant et remplaçant toutes dispositions antérieures contraires notamment la directive 05-97 du 16 décembre 1997 relative aux lois de finances et ses textes modificatifs. Voir article 87 de la directive 06-2009.

28 MBODJ (F), Revue « droit sénégalais » $\mathrm{n}^{\circ} 7$ novembre 2008; page 317 et suivant, Presse de 1’Université des Sciences de Toulouse. 
pour laquelle, il est érigé au rang de principe. C'est-à-dire élevé en une proportion admise comme base de science ou d'un raisonnement.

C'est ainsi que Loïc Philip définit les principes budgétaire comme « les principes juridiques de droit public qui gouvernent la vie d'un budget pris au sens juridique du terme. Ils concernent donc : l'Etat, les collectivités locales, les établissements publics administratifs et les communautés européennes... $»^{29}$. C'est ainsi qu'en droit budgétaire local, le principe de l'équilibre présente une spécificité dès lors que les textes qui encadrent le budget local insistent sur caractère réel de l'équilibre. D'où la portée obligatoire pour les collectivités locales.

En effet les collectivités locales sont assujetties à une obligation de voter leurs budgets en équilibre. La loi organique ${ }^{\circ} 2001-09$ du 15 octobre 2001 relative aux lois de finances met à la charge des CL une obligation de voter en équilibre leurs budgets et en parfaite harmonie avec le droit communautaire. Cette obligation légale est confirmée par la loi $\mathrm{n}^{\circ}$ 2013-10 du 28 décembre 2013 portant code général des collectivités locales. C'est la combinaison des articles 255 et 256 qui renseigne de la réalité du caractère obligatoire du principe d'équilibre du budget local. En effet l'article 255 n'utilise dans sa définition de budget local, ni le conditionnel, ni même l'imparfait mais un temps qui exprime bien l'obligation et la réalité, qui est le présent de l'indicatif. Toutefois, le caractère obligatoire de l'équilibre budgétaire est porté par l'article 256 qui dit « lorsque le budget n'est pas voté en équilibre réel... », le représentant de l'Etat le règle à partir d'une procédure particulière et le met en œuvre.

Un autre type de texte plus près, moins connu et peu familier de certains acteurs des finances locales, vient renchérir cette obligation en posant lui aussi l'obligation pour les CL de voter le budget en équilibre réel. Il s'agit du décret $n^{\circ} 66-510$ du 04 juillet 1966 portant régime financier des $\mathrm{CL}^{30}$.

Le texte législatif en son article 255 pose que « Le budget d'une CL est en équilibre réel lorsque « la section fonctionnement » et « la section investissement » sont respectivement votées en équilibre, les recettes et les dépenses ayant été évaluées de façon sincère, et lorsque les prélèvements sur la section fonctionnement au profit de la section investissement, ajouté aux recettes propres de cette section, à l'exclusion du produit des emprunts et éventuellement aux dotations des comptes d'amortissement et de provision fournit des ressources suffisantes pour couvrir le remboursement en capital des annuités d'emprunt à échoir au cours de l'exécution $»^{31}$. Mais cette définition longuement détaillée de l'article $255 \mathrm{du}$ CGCL reste évasive quant aux contours et au contenu de l'équilibre du budget local. Est-il un équilibre fondé sur une adéquation conforme entre section? Ou bien, est-il un équilibre comptable entre sections?

29 Voir, DERUEL (B), Budget et pouvoir financier, $10^{\text {ème }}$ édition, $p, 133$ et suivants.

30 Décret nº6-510 portant régime financier des collectivités locales, JO du 23 juillet 1966, p.891 et suivants.

31 Loi 2013-10 du 28 décembre portant Code général des CL, article 255 du Code général des CL. 
L'article 07 du décret $n^{\circ} 66-510$ du 04 juillet 1966 portant régime financier des CL a le mérite d'être plus exhaustif. Aux termes des dispositions de cet article « les prévisions de recettes de la section ordinaire doivent être au moins égales aux prévisions de dépenses de cette section; les prévisions de dépenses de la section extraordinaire ne peuvent être supérieures aux prévisions de recettes de la section qu'au cas où la section ordinaire fait apparaître un excédent de recettes et à dans les limites de cet excédent; l'équilibre de la section ordinaire ne peut, en aucun cas, être assuré par un prélèvement sur la section extraordinaire $»^{32}$.

Ce régime quoi que plus exhaustif et plus clair ne contredit pas celui posé à l'article 255 du CGCL. C'est ainsi que la combinaison de ces deux articles s'avère pertinent et permet de déceler deux régimes par déduction. Il s'agit d'un régime " de conditions à remplir » (excédent de la section ordinaire et déficit de la section extra ordinaire) et un régime « d'interdits » (jamais de prélèvement sur la section extra ordinaire au profit de la section ordinaire $)^{33}$. Mais à la question de savoir si ces conditions sont alternatives ou cumulatives, la seconde proposition prime sur la première.

Il est très important de rappeler que cet équilibre tient son caractère multiforme des différents documents budgétaires à partir desquels il peut s'apprécier.

Dans un premier temps, rien qu'au sein du même budget primitif, l'équilibre se note par section. C'est un équilibre comptable et interne car requérant une égalité entre recettes d'investissement et dépenses d'investissement, mais aussi entre recettes de fonctionnement et recettes de fonctionnement. Il y a en outre un équilibre externe ou global. A ce niveau les recettes totales sont égales aux dépenses totales. Mai il faut, comme le fait remarquer le docteur Fara Mbodj, dire que cet équilibre respectif entre sections auquel renvoie l'équilibre comptable est absent des dispositions du décret 66-510 du 04 juillet 1966 portant régime financier des CL. Mais l'équilibre comptable n'épuise pas les caractères de l'équilibre du budget local. En effet l'équilibre a également une dimension financière. Il s'agit de l'équilibre financier qui décrit une balance entre ressources et charges de la CL, il en découle forcément un équilibre susceptible de constituer un équilibre prévisionnel ${ }^{34}$.

Cette dimension financière a été insinuée dans les dispositions finales de l'article 255 du CGCL dans lequel il est appelé « mouvement financier ». Toujours, le caractère multiforme de l'équilibre budgétaire est aussi apparent au niveau du budget supplémentaire. Sa définition est explicite, en effet, c'est un document qui sert à rééquilibrer les prévisions du budget primitif et intervient en cours d'exécution ${ }^{35}$. L'équilibre s'apprécie aussi au niveau

32 Décret n66-510 du 04 juillet 1966 portant régime financier des CL, p. 892.

33 Notons que la section ordinaire correspond à la section fonctionnement, et la section extra ordinaire correspond à la celle investissement. Voir article 5 du décret 66-410 du 04 juillet 1966 précité.

34 MBODJ (F), Revue « droit sénégalais » ${ }^{\circ} 7$ novembre 2008; page 317 et suivant, Presse de 1’Université des Sciences de Toulouse.

35 DIAGNE Abdou Aziz, mémoire de DEA de décentralisation et de gestion des collectivités locales, «Contrôle des collectivités locales par le représentant de l’Etat 1996-2005 », 2005, p. 68. 
des autorisations spéciales qui décrivent toutes les opérations nouvelles ou réévaluation qui seraient apparues nécessaire depuis l'établissement du budget. Elles doivent aussi être équilibrées quelque soit la section à laquelle elles pourront être rangées.

Elaboré par l'exécutif local et voté par l'assemblée délibérante, le compte administratif retrace toutes les recettes et dépense réalisées au cours de l'année y compris celles qui ont été engagées et non payées ${ }^{36}$. Il donne donc une idée sur la manière dont le budget a été exécuté et les résultats de l'exécution comparant recettes et dépenses. Il permet donc de contrôler l'équilibre d'exécution. Quant au compte de gestion, il est établi par le comptable de la CL et retrace les flux de recettes et de dépenses effectivement réalisées au cours de l'année budgétaire. De ce fait il permet, par le truchement du comptable, de contrôler l'équilibre du budget de la collectivité locale. La correspondance entre les recettes et les dépenses réalisées renseigne la Cour des comptes de l'équilibre du budget au moins pour les flux déjà exécutés.

Cependant, multiforme, le principe de l'équilibre pourrait être très difficile à appréhender. C'est dans ce sens qu'il a été érigé une règle d'ordre conceptuel guidant et éclairant l'équilibre budgétaire local. Il 'agit du principe de sincérité. En réalité la sincérité donne à l'équilibre son caractère réel. Autrement dit, l'équilibre réel du budget local renvoie à une tâche d'élaboration des recettes et des dépenses qui renoncent aux aléas et à la démarche approximative. Il s'agi alors d'une démarche guidée par le principe de sincérité.

En claire, la sincérité ne permet de « ne faire figurer dans le budget local que les recettes dont le principe est acquis. Toute inscription de ressources fictives ou abusivement évaluées ne peut que conduire à l'aggravation du déséquilibre» ${ }^{37}$.

Les dépenses doivent également être évaluées de manière réaliste. Il s'agit d'une appréciation véridique des recettes et des dépenses qui intègre largement l'éthique financière. Autrement dit le principe de sincérité interdit la surévaluation des recettes, la surestimation ou la sous estimation des dépenses. Ce principe est consacré par l'article 255 du CGCL qui énonce que « les recettes et dépense ayant été appréciées de façon sincère ${ }^{38}$

Selon le professeur Muzellec : «Le principe de sincérité est à la fois un principe comptable et budgétaire qui gouverne la préparation et la présentation de la loi de finances. Il se ramène à la bonne foi qui implique une évaluation aussi correcte que possible» ${ }^{39}$.

C'est dans cette perspective que des méthodes d'évaluation fiables sont envisagées pour donner une effectivité au principe de sincérité. A cet égard, il convient d'en citer quelques unes. Spécifique les unes par rapport aux autres, ces méthodes peuvent être combinées pour donner lieu à des méthodes mixtes ou corrigées pour déterminer le contenu des principaux postes.

36 Article 259 du CGCL.

37 CISSE (MD) et DIOUF (MP), Manuel de procédure de contrôle de légalité et budgétaire, octobre, 2001, op.cit.

38 Loi 2013-10 du 28 décembre 2013 portant général des collectivités locale, p. 34 et s.

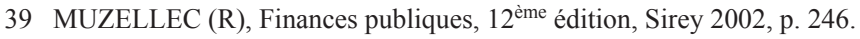


- La méthode de la pénultième année : Elle consiste à effectuer les calcules des postes en tenant compte des résultats de la gestion précédente. Cette méthode présente de sérieuses difficultés. En effet, au moment de l'élaboration du budget, la situation financière de la CL n'est pas encore définitive. Il s'y ajoute que la situation d'un poste peut varier d'une année à une autre.

- La méthode d'évaluation directe : Elle consiste à effectuer certaines prévisions à partir de l'existence et du taux de la taxe.

- La méthode de la moyenne triennale : Elle consiste à prendre pour chaque poste, la somme des réalisations des trois dernières années connues et en divisant cette somme par le nombre d'année. On obtient la moyenne représentant la prévision de la prochaine gestion. Aléatoire! Voilà le qualificatif qui sied.

- La méthode des projections budgétaires: Celle-ci considère le taux d'évaluation des réalisations de chaque type de recettes ou de dépenses calculé pendant les 3,4 ou 5 dernières années qu'on applique aux réalisations de la dernière gestion.

- Le budget à base zéro : C'est une technique de prévision de dépenses et de recettes qui se fonde chaque fois que c'est possible sur des fichiers constitués par les services d'assiette de la CL. Cette technique est souvent appliquée aux CL nouvellement instituées qui ne disposent pas alors de statistiques sur les années précédentes.

- La méthode combinée : Avec cette méthode les prévisions obtenues par l'une ou l'autre méthode doit être corrigées par :

- Les données réelles fournies par les dégrèvements et exonérations.

- Les possibilités de recouvrement de la CL (le taux de recouvrement réel).

- Les informations fournies par une comptabilité administrative à jour.

- Les décisions et orientations prises par la CL dans ses politiques fiscales.

Toutefois, ce principe étoffé et enrichi par le principe de sincérité s'avère à certains endroits pesant. Ainsi, est-il important de souligner l'existence de certaines inflexions.

Le principe de l'équilibre réel du budget local renvoie à un dispositif matériel au sens des règles du droit budgétaire en général et local en particulier. Ce dispositif est contraignant dès lors qu'il a une portée obligatoire pour les CL. Toutefois, il convient de faire remarquer que la rigueur originelle qui s'attache au principe peut entacher son effectivité. C'est pourquoi des inflexions se sont montrées nécessaires.

Ces inflexions sont analysées dans le sens d'une exception et dans le sens d'un aménagement.

Dans un premier temps, disons que l'exception de l'équilibre du budget local est révélée par l'hypothèse d'un budget excédentaire.

Le budget excédentaire est caractérisé par une supériorité des recettes sur les dépenses. Cette situation quasi exceptionnelle avait une signification péjorative dans l'ère des finances publiques classiques. En effet, l'excédent budgétaire était toujours appréhendé comme un prélèvement injustifié symbole d'une mauvaise gestion. 
Aujourd'hui, l'excédent budgétaire a été réhabilité et retrouve des vertus de bonne gestion financière économique. Il est très recherché ${ }^{40}$. Pourtant, dans le droit budgétaire local, l'excédent est aménagé comme une exception, le droit commun étant l'équilibre budgétaire. L'explication de ce paradoxe est à rechercher dans la logique d'une orthodoxie financière et comptable. En effet, des risques certains existent en voulant infléchir le principe de l'équilibre budgétaire dans un sens ou dans l'autre pour des personnes morales qui ne disposent d'aucune autonomie dans la détermination des masses de dépense les plus importantes, mais aussi d'aucune autonomie dans la perception des recettes ${ }^{41}$.

C'est alors toute la problématique de l'hypothèse d'un budget excédentaire dans les CL du Sénégal. Par exemple, en matière fiscale, les impôts locaux représentent plus de $60 \%$ des recettes budgétaires des communes mais les communes ne déterminent ni les taux ${ }^{42}$, ni les assiettes ni même les modalités de recouvrement des impôts locaux.

Si le décret 66-510 du 04 juillet 1966 reste silencieux à propos de l'hypothèse d'un budget excédentaire, c'est une hypothèse retenue néanmoins par le CGCL. En effet, l'article 257 du CGCL pose que « Toutefois, pour l'application de ces dispositions, n'est pas en déséquilibre, le budget dont la section fonctionnement comporte ou reprend un excédent et dont la section investissement est en équilibre réel, après reprise, pour chacune des sections, des résultats apparaissant au compte administratif de l'exercice précédent $»^{43}$. La portée d'une telle disposition se ramène à l'hypothèse d'excédent budgétaire circonscrit exclusivement à la section fonctionnement. En réalité la section investissement doit être en équilibre parfaite.

Donc, l'hypothèse d'un budget local excédentaire est admise par la loi, mais délimitée à la section fonctionnement. Cela veut dire que les recettes de fonctionnement peuvent être supérieures aux dépenses de fonctionnement.

Autrement dit, c'est le résultat qui décrit un solde excédentaire conçu comme la manifestation d'un vote du budget local en déséquilibre par excès de recettes sur les dépenses de la même section fonctionnement.

C'est en cela que l'hypothèse d'un budget excédentaire constitue une exception au principe de l'équilibre réel du budget local qui reste le droit commun en matière d'élaboration et d'exécution du budget.

Mais dans la mesure où la section fonctionnement se fait le terrain de pratiques décrites par l'opinion parce que prévoyant des dépenses de fonctionnement faramineuses et démesu-

40 D'ailleurs en France, avec la loi de du 5 février 1988, la règle de l'équilibre n'est plus absolue. Avec cette dite loi, seul le déficit est strictement interdit. Depuis cette date donc, l'inscription d'excédent en section fonctionnement n'est plus un motif de déséquilibre.

41 L'article 67 de la Constitution 2001-09 du 22 janvier 2001cite dans la catégorie des matières réservées au domaine de la loi « l'assiette, le taux et les modalités de recouvrement des impositions de toutes natures... ».

42 Les CL peuvent, bien attendu, se mouvoir par leurs délibérations entre un taux planché et un taux plafond. La taxe rurale par exemple varie entre 500 et 1000F Cfa.

43 Loi 2013-10 du 28 décembre 2013 portant général des collectivités locale. 
rées $^{44}$, il est pertinent de se poser des questions sur l'opportunité ou la rationalité d'une telle exception. Aussi, la pauvreté de la quasi-totalité des CL du Sénégal, assortie des politiques budgétaires et fiscales de l'Etat donne à cette exception un caractère surréaliste et irrationnel.

A côté de cette exception, la loi aménage le principe de l'équilibre réel du budget à partir de ce qu'il est convenu d'appeler « pudiquement» le mouvement financier qui s'analyse comme un artifice comptable.

Dans un second temps, nous dirons que le mouvement financier nous fait passer d'un équilibre réel à un équilibre virtuel. Absent du dispositif du décret 66-510 du 04 juillet 1966 portant régime financier des CL, le mouvement financier est apparu à l'article 255 de la loi de 2013 en ses termes : «Lorsque le prélèvement sur les recettes de la section fonctionnement au profit de la section investissement, ajouté aux recettes propres de cette section, à l'exclusion des produits des emprunts, et éventuellement aux dotations des comptes d'amortissement et de provision fournit des ressources suffisantes pour couvrir le remboursement de la dette en capital des annuités d'emprunt à échoir au cours de l'exercice... ${ }^{45}$. Il s'agit d'une articulation entre les deux sections emportant une migration de crédits. Il s'applique dès lors que les prévisions de dépenses de la section d'investissement sont supérieures aux prévisions de recettes de la section d'investissement. Ce n'est qu'en ce moment que l'on écrête le surplus de ces recettes de la section fonctionnement au profit de la section investissement qui se trouve déficitaire.

En des termes plus simples, ce mouvement financier décrit véritablement un équilibre prévisionnel attaché à trois conditions cumulatives : il faut d'abord qu'il y est un excédent de la section fonctionnement. Il faut ensuite un déficit de recettes de la section d'investissement. Il faut enfin un ordre de recette et une opération d'ordre au nom du receveur municipal.

A en croire à M. Fara Mbodj, un regard dans les documents budgétaires révèle un recours démesuré et suspect au mouvement financier en vue d'un équilibre prévisionnel. Il poursuit que « ce recours obsessionnel à cet artifice comptable fait penser que l'équilibre réel du budget local constitue un véritable mythe ». Il s'analyse alors comme un ensemble de croyances bâties autour d'un fort sentiment d'attraction. En effet, le recours au mouvement financier déroule une tentative d'explication et de solution sommaire à partir d'un critère délibérément exagéré : l'excédent. ${ }^{46}$ En réalité, toute une stratégie conceptuelle et comptable est développée dans le but d'atteindre un équilibre hypothéqué d'avance compte tenu d'un environnement économique et financier défavorable. Dans cette perspective, la quête d'un équilibre budgétaire est illusoire. C'est le mythe de l'équilibre réel du budget.

44 Surtout pour ce qui concerne le chapitre 313 relatif aux dépenses de fonctionnement afférant au cabinet du maire.

45 Article 255 de la loi 2013-10 du 28 décembre 2013 portant général des collectivités locales.

$46 \operatorname{MBODJ}(\mathrm{F})$, Revue « droit sénégalais » $\mathrm{n}^{\circ} 7$ novembre 2008; page 317 et suivant, Presse de 1’Université des Sciences de Toulouse. 
Cependant, au risque de faire fie de la signification de l'équilibre budgétaire au niveau des personnes morales de droit public chargées de la gestion d'un service public, nous verrons donc comment ce principe d'équilibre budgétaire est-il entendu dans les services personnalisés de l'Etat et qui sont dotés de budget autonome, à savoir les établissements publics ${ }^{47}$. Ne pouvons nous pas dire d'emblée que cet équilibre est souhaitable?

A travers la jurisprudence du conseil constitutionnel, on peut définir l'établissement public selon quatre éléments. D'abord, la personnalité juridique. Il est juridiquement autonome, il dispose de droits et d'obligations (prendre des actes, avoir un patrimoine et un budget propre par exemple). Ensuite, la gestion d'activité d'intérêt général. L'établissement public doit fournir des prestations d'intérêt collectif et doit exercer une activité de service public (administratif ou industriel et commercial). Puis, le rattachement à une collectivité territoriale. Cela signifie qu'ils sont créés soit par l'Etat, soit par une collectivité locale et durant toute leur vie, ils sont soumis à la tutelle de la collectivité de création. La tutelle est administrative (nomination des membres au sein du conseil d'administration) et financière (le budget des établissements publics administratifs est souvent alimenté totalement par leurs collectivités créatrices). Enfin, la soumission au principe de spécialité, ici leurs compétences sont limitativement énumérées (exemple : hôpitaux et universités).

Ce principe fixé par la loi entre en contradiction avec la clause générale de compétences dont bénéficient les collectivités locales et qui leur permettent d'intervenir dans de très nombreux domaines de la vie locale ${ }^{48}$.

Cependant, la question est de savoir comment se conçoit le budget de l'établissement public en tenant compte du principe de l'équilibre budgétaire qui n'épargne pas leurs finances. En d'autres termes, est-il permis un déficit ou un excédent du budget desdits établissements publics administratifs?

Il faut dire d'emblée, que ces établissements publics disposent de budget autonome qui, au sens strict du mot autonome, désigne les budgets des personnes publiques distincts de l'Etat et dont les comptes prévisionnels ne sont pas intégrés dans la loi de finances de l'année.

La confection des budgets des établissements publics administratifs est donc, peu ou prou, astreint au principe de l'équilibre. En effet, dans la mesure où l'équilibre économique et financier d'un Etat est jaugé tant dans le budget de l'Etat que dans ceux des Collectivités locales et des Etablissements publics ainsi que toutes les personnes publiques qui interviennent dans les secteurs socio économiques comme les entreprises publiques. Aussi, l'État garantit la solvabilité de tous les établissements publics nationaux et les prémunit contre tout risque d'insuffisance de crédit. En France, on a la Caisse d'Amortissement Des Etablissements Sociaux (CADES) qui a pour mission d'amortir la dette sociale française au moyen d'une structure financière équilibrée, s'appuyant notamment sur des ressources fis- 
cales. Elle est une institution, qui allie les garanties que peut offrir un établissement public (par exemple l'impossibilité de faire faillite).

Au Sénégal, en tant qu'établissement public ayant fait l'objet d'un contrôle par la cour des comptes on peut prendre comme exemple du Centre National Appareillage Orthopédique (CNAO) qui a porté sur les gestions 2002 à 2005 et a donné lieu à des observations et recommandations. La cour se limite à dire que les budgets exécutés pour les exercices 2005 et 2006 n'ont fait l'objet ni d'approbation, ni d'un suivi et d'un contrôle satisfaisants ${ }^{49}$.

Mais n'est-il pas intéressant de faire remarquer que dans EPIC le déséquilibre toléré. Un établissement public caractère industriel et commercial (EPIC) est une personne publique ayant pour but la gestion d'une activité de service public et ayant une autonomie financière. Les EPIC ont été créés pour faire face à un besoin ne pouvant pas être correctement pris en charge par une entreprise privée soumise à la concurrence. Les EPIC aussi répondent au principe de la spécialité. L'activité de l'établissement est limitée au service public qu'il a pour mission de gérer. Ainsi, il ne peut pas employer ses biens et son patrimoine à d'autres activités, sauf si celles-ci concourent, même indirectement, au service public qui lui est confié. De même, il ne peut accepter que des dons correspondant à sa mission. Les établissements publics sont rattachés à l'État ou à une collectivité territoriale de tutelle.

Comme toute personne morale, l'établissement public possède des biens matériels. Ainsi, son autonomie se traduit essentiellement par l'existence d'un budget autonome, détaché du budget général de la collectivité dont il dépend. Mais à la question de savoir si leur budget répond au principe de l'équilibre, la réponse doit sans doute être mitigée. En effet, leur budget est alimenté par des ressources propres qui sont notamment constituées des produits d'exploitations tirés des services rendus contre rémunération, des libéralités et des emprunts.

Mais pour un budget qui est censé être équilibré, on ne recourt à l'emprunt que si les prévisions de dépenses sont plus importantes que les prévisions de recettes. Donc, avec la possibilité d'emprunt offerte, on sent un parfum de déséquilibre budgétaire des EPIC. La justification pourrait être tirée de son objet et sa nature industrielle et commerciale. Ainsi, toutes les astuces permettant de concilier l'indispensable recherche de l'intérêt général et l'inévitable quête de bénéfices, sont dès lors utilisables à condition qu'elles ne soient pas contraires aux principes qui régissent l'établissement public.

De ce fait, suffit-il de poser un cadre juridique applicable à tous les niveaux? Il nous semble plus opportun de poser aussi des garanties qui assurent la fiabilité dudit principe pour en récolter tous avantages qu'on lui prête. 


\section{$I^{\circ}$ ) Les garanties de l'effectivité du principe de l'équilibre budgétaire.}

Pour garantir efficacement l'effectivité d'un principe, il ne suffit pas de le poser, il faut aussi l'adosser à un système de contrôle (A), mais nous ne manquerons pas d'en étudier la portée (B).

A - Le contrôle de l'équilibre budgétaire.

La garantie la plus sûre s'avère être le contrôle. Le critère de distinction mis en exergue dans le contrôle du principe de l'équilibre budgétaire est le caractère interne ou externe de ces contrôles. Ainsi, aura-t-on d'une part les contrôles administratif et comptable et d'autre part les contrôles politique et citoyen.

Il est important de préciser d'emblée que le contrôle de l'équilibre budgétaire satisfera les attentes portées en lui, plus en ce qui concerne les finances locales que dans les finances étatiques. En effet, obligation légale pour les collectivités locales, le principe de l'équilibre est " plus qu'un vœu et moins qu'un ordre ${ }^{50}$ pour l'Etat dans le cadre de l'union car son irrespect n'est conséquence d'aucune sanction ${ }^{51}$.

C'est dans ce cas là qu'on peut affirmer que ce contrôle est plus important dans les budgets des autres entités personnalisées de 1'Etat surtout des collectivités locales. Néanmoins, nous réserverons des développements concernant les budgets des collectivités locales et des établissements publics.

S'agissant de l'équilibre des collectivités locales, le contrôle administratif y est pertinent. Cela découle de l'une des conditions d'existence de la décentralisation, à savoir le contrôle de l'Etat. Ici, comme dans bien d'autres domaines de la décentralisation, le représentant de l'Etat joue un rôle prépondérant. En effet le contrôle administratif dont il s'agit ici, s'entend essentiellement du contrôle exercé par le gouverneur, le préfet ou le sous-préfet. Ils sont des autorités déconcentrés du pouvoir central. Ils exercent une mission de suivi et de contrôle auprès des collectivités locales pour le compte de l'Etat. Ce contrôle plus présent et plus pesant s'explique et se justifie par l'unité de la mission de l'Etat, mais aussi et surtout par la rareté et la nécessité qui caractérise les ressources publiques. Il s'y ajoute que les collectivités locales procèdent à des dépenses publiques. Et comme l'a si bien rappelé les professeurs Laferrière et Waline à l'occasion de l'affaire du Mont de Piété de Bayonne « Le déséquilibre du budget n'est pas unes affaire locales : l'Etat est considéré par l'opinion

50 Termes empruntés à Martine Lombard dans son ouvrage «droit administratif », $3^{\text {ème }}$ édition Dalloz.

51 Exemple de la France à la suite de son déficit cyclique de plusieurs années consécutive au sein de l'UE malgré la réglementation sans équivoque qui autorise un déficit inférieur ou égal à $3 \% \mathrm{du}$ PIB de chaque Etat. Récemment, la Grèce rappelée à l'ordre mais cette fois ci à vive voix. Ce qui a abouti à l'adoption d'un plan d'austérité. 
comme responsable de la gestion financière des collectivités locales; l'effet politique des mauvaises gestions communales peut être désastreux $\gg .52$

Il existe un autre argument purement budgétaire qui est le principe de l'autorisation de percevoir les recettes et d'effectuer les dépenses ${ }^{53}$. A cet effet, le contrôle budgétaire vise à assurer la conformité des opérations budgétaires à l'acte d'autorisation conféré par l'organe délibérant.

Le représentant de l'Etat a des « pouvoirs » gradués compte tenue de la matière qui doit faire l'objet du contrôle. Il dispose de « compétences » étendues pour les matières qui entrent dans le champ d'application de l'article 336 du code des collectivités locales. Cet article renvoie au budget générique, aux actes de planification dans le temps et dans l'espace, aux contrats les plus importants etc.

Ainsi dans le cadre budgétaire, l'étendu des attributions du RE est mise en exergue par son pouvoir d'approbation du budget de la CL. Le RE veille à l'équilibre réel du budget sous la dimension comptable et financier prévue à l'article 255 du CGCL ${ }^{54}$. Il sanctionne aussi le système de compensation décrit à l'article 07 du décret 66-510 du 04 juillet 1966 interdisant formellement que l'équilibre de la section « fonctionnement » soit assuré par des prélèvements de la section « investissement $»^{55}$.

L'inobservation des conditions d'existence d'un équilibre réel est sanctionnée par le refus par le RE, d'approuver le budget de la CL en question. Mieux, la loi lui permet, dans certaines circonstances, de régler le budget et de le rendre exécutoire ${ }^{56}$.

Toujours, il appartient au RE de contrôler le compte administratif de l'ordonnateur du budget local (président de l'exécutif local). Les RE veillent à l'équilibre du budget de la CL en sanctionnant tout déficit supérieur ou égal à $10 \%$ des recettes de la section fonctionnement.

Mais ce contrôle, aussi " lourd » soit il, n'entame en rien celui que peut exercer les autres organes de contrôle tels que l'Inspection Générale d'Etat ${ }^{57}$ (IGE) le Contrôle Finan$\operatorname{cier}^{58}(\mathrm{CF})$ et l'Inspection Général des Finances (IGF) $)^{59}$.

Ils ont compétence à contrôler toutes les opérations financières de l'Etat, des établissements publics, des régions, communes, et communautés rurales, des sociétés nationales et des personnes morales bénéficiant du concours financier de l'Etat. Le contrôle de l'IGE par exemple est d'ordre général. Il porte sur l'efficacité, l'opportunité mais surtout sur la légali-

52 LAFERRIER $(\mathrm{J})$ et WALINE $(\mathrm{M})$, « Traité élémentaire de science et de législation financière », LGDJ, 1951, p. 191.

53 Article 68 de la constitution du 22 janvier 2001 op. cit.

54 Loi 2013-10 du 28 décembre 2013 portant général des collectivités locales op. cit.

55 Décret numéro 66-510 du 4 juillet 1996 portant régime financier des collectivités locales op. cit.

56 Article 264 de la loi 2013-10 du 28 décembre 2013 portant général des collectivités locales.

57 Décret numéro 80-914 du 5 septembre 1980 portant organisation de l'IGE.

58 Décret numéro 78-85 du $1^{\text {er }}$ Février 1978 portant organisation du CF.

59 Dépend directement du Ministre de l'Economie et des Finances. 
té. C'est cette légalité et le champ géographique illimité de son pouvoir de contrôle qui lui donne effectivement compétence à contrôler, justement, l'équilibre des budgets des CL. Mais il faut noter que dans le cadre de 'équilibre, ce contrôle ne sera que résiduel ${ }^{60}$. C'est pourquoi dans le cadre des tournées inopinées de ladite IGE le point est généralement mis sur l'opportunité et l'efficacité de l'emploi de deniers publics.

S'agissant du contrôle comptable, il et exercé par tout fonctionnaire ou agent ayant qualité pour exécuter au nom d'un organisme public des opérations de recettes, de dépenses de maintien des titres... ${ }^{61}$ Le comptable public est un agent séparé de l'ordonnateur sur le fondement de la règle de séparation des comptables et des ordonnateurs. Au niveau étatique le contrôle de l'agent comptable n'est pas sévère du tout en ce qui concerne l'équilibre du budget. Ceci tient au fait que l'interdiction faite aux Etats de voter leur budget en déséquilibre est neutralisée dès le vote de ce qu'il est convenu d'appeler «l'article d'équilibre » et qui confère au budget de l'Etat une légalité formelle.

Au niveau de la CL, le comptable exerce deux fonctions : payeur et caissier. Le problème qui se pose est de savoir si un comptable peut exécuter des opérations de recettes et de dépenses tirées d'un budget en déséquilibre?

Le premier palier que constitue le contrôle du RE devait, comme rappelé tout à l'heure, vider le contrôle du budget de tout sens au-delà. Cet écueil contourné par quelle que puisse être la raison, liera-t-il le comptable public? Autrement dit, le comptable doit-il recouvrer les recettes et exécuter les dépenses de la CL dont le budget est voté et approuvé en déséquilibre.

Cas pratique vraisemblable! Devant cette hypothèse, on considérera d'bord que le comptable comme tout agent de l'administration est soumis à la légalité. Ainsi, ses opérations de recettes et de dépenses doivent être conformes au droit en général. La loi de 2012 stipule clairement que « le budget de la collectivité locale doit être voté en équilibre réel », alors rien que sur ce fondement, le comptable peut valablement refuser d'exécuter des opérations de recettes et de dépenses. En effet, si le comptable ne peut subordonner ses actes de paiement à l'appréciation de l'opportunité des décisions prises par l'ordonnateur, il peut néanmoins les soumettre à un contrôle de légalité au prix de sa responsabilité personnelle et pécuniaire. La tenue du compte de gestion par le comptable inspire fortement l'édiction du compte administratif tenu par le chef de l'exécutif local. C'est ainsi que le comptable, par son compte de gestion est lui-même « jauge » de l'équilibre du budget de la CL dont il tient les deniers et titres. En termes plus clairs, il est possible de procéder au contrôle de l'équilibre budgétaire de la CL rien qu'en consultant le compte de gestion tenu par le comptable de cette dernière. Car ce compte recèle les flux de recettes effectivement exécutées en cours d'année.

60 Ce contrôle ayant été vidé par le RE avant la mise en exécution du budget.

61 Loi 2012-23 du 23 du 27 décembre 2012 abrogeant et remplaçant la loi nº 99-70 du 17 février 1999 sur la Cour des Comptes. 
Il est important de signaler le contrôle non juridictionnel de la Cour des Comptes. En effet, le contrôle de la Cour des Comptes est un contrôle global qui saisit l'ensemble des aspects de la gestion budgétaire. Il lui est loisible, au plan local, de s'appesantir sur l'équilibre réel du budget, et son corollaire, la sincérité des comptes administratifs et des comptes de gestion. Il convient de rappeler que cette forme de contrôle non juridictionnel reste l'outil le plus adapté pour exercer un droit de regard sur la gestion de la CL. C'est un moyen d'atteindre l'ordonnateur par l'examen de sa gestion. Le contrôle se fait sur pièce et sur place et reste un moyen de contrôler l'équilibre réel du budget et de sa sincérité.

Mais il existe d'autres types de contrôle, cette fois-ci plus externes que les contrôles administratif et comptable, il s'agit des contrôles politique et citoyen.

Contrôle politique se confond ici avec contrôle des assemblées. Ce peut être le Parlement pour l'Etat ou le conseil régional pour la région, le conseil municipal pour 1 commune ou le conseil rural pour la communauté rurale. Mais, dans le long processus budgétaire qui va de la préparation au contrôle en passant par le vote et l'exécution, on constatera que les assemblées ne sont effectivement impliquées qu'avant la mise en exécution et de manière très timide après celle-ci. Ainsi comment le Parlement ou l'organe délibérant de la collectivité locale exerce t-il un contrôle sur l'application du principe de l'équilibre?

En ce qui concerne le Parlement et le contrôle de l'application du principe de l'équilibre du budget de l'Etat, en l'absence de dispositions législatives et règlementaires interdisant de manière formelle que le budget de l'Etat soit voté en déséquilibre, on rappellera le contenu de l'article 98 de la constitution selon lequel les traités régulièrement ratifiés ont dès leur publication une autorité supérieure à celle de la loi ${ }^{62}$. Ceci assorti de l'adhésion formelle faite par l'Etat du Sénégal au traité de l'UEMOA du 14 novembre 1976, fait des directives prises par le Conseil des Ministres de l'union des directives « directement applicables » ${ }^{63}$. De ce simple fait, le Parlement devra en tenir compte au moment de l'adoption du budget.

Il faut dire à ce stade que le Parlement lui-même participe au contournement de ce principe en autorisant le Président de la République à recourir à l'emprunt afin de couvrir formellement le déficit du budget.

Dans l'hypothèse où le déficit dépasserait 3\% du PIB aussi, la menace de voir tous les services de l'Etat en éclipse justifierait le vote du budget par les Assemblées. C'est d'ailleurs cette limite ajoutée à l'indiscipline budgétaire des Etats membres qui a conduit à l'adoption de l'Acte additionnel n 04-99 du Conseil des Ministres portant Pacte de convergence, de stabilité, de croissance et de solidarité entre Etats membres de l'UEMOA. Mais, la souplesse de ce pacte tient à ce que le déficit n'est réprimé que s'il fausse les grandes lignes de l'équilibre des pays membres de l'union. En d'autres termes, tant que la stabilité économique n'est pas secouée, le pays en question n'est que verbalisé.

62 Constitution du Sénégal du 22 janvier 2001.

63 Les directives de l'union lient tout Etat membre, mais laissent la compétence aux instances nationales quant à la forme et aux moyens, Voir CORNU (G), Vocabulaire Juridique, PUF, 1998. 
Mais le Parlement dispose aussi d'un pouvoir de contrôle a posteriori qui est l'examen de la loi de règlement. La loi de règlement est un instrument de verrou mis à la disposition des parlementaires. En effet, pour permettre à la représentation populaire d'exercer un contrôle efficace sur la gestion des FP, la directive du 26 juin 2009 relative aux Lois de Finances dispose que : « les projets de loi de règlement doivent être obligatoirement déposés au bureau de l'Assemblée Nationale au plus tard le 31 Décembre de l'année suivant celle de l'exécution du budget concerné... " ${ }^{64}$ Cet examen de la LR est loin d'être sans objet, en ce sens son intérêt dépasse la juste vérification du budget déjà réalisé pour se préoccuper des budgets prochains afin d'en apporter les rectificatifs idoines le cas échéant.

Le principe de l'équilibre budgétaire est un « principe de prévision » et non " d'exécution » c'est-à-dire que sa mise en œuvre se fait avant ou au moins au moment de l'établissement du budget. Contrairement aux budgets des CL, l'équilibre du budget de l'Etat n'est pas soumis à une obligation légale. Ce qui aurait été, en réalité, plus contraignant qu'une interdiction découlant d'une directive de l'union.

Dans ce sillage même, la directive précitée prévoit que si l'exigence du dépôt de la LR n'est pas respectée, le Parlement ne procède pas au vote de la loi de finances de l'année à $v^{v e n i r}{ }^{65}$. Combien de fois avons nous constaté un retard cyclique de plusieurs années pour le dépôt de la LR? Et dans la foulée, le parlement vote le budget de l'année à venir, illustration du caractère facultatif du principe de l'équilibre dans l'Etat. Il faut quand même noter des efforts dans la résorption de ce gap depuis quelques années.

Concernant, le contrôle de l'équilibre des budgets des CL, son assise juridique est plus palpable. En effet, il s'agit d'une obligation légale pour la CL de voter son budget en équilibre réel posée par le CGCL. Ainsi quel est le rôle du comptable public de la CL dans ce contrôle?

En réalité, le principe de l'équilibre réel du budget local saisit les opérations budgétaires du budget primitif, du budget supplémentaire et de toutes les décisions modificatives de nature budgétaire. D'ores et déjà, il convient de signaler que toujours avant l'exécution du budget, il existe un débat d'orientations budgétaires qui constitue un espace de contrôle des comptes de la CL. D'ailleurs ce débat reste une obligation légale aux termes des dispositions de l'article 253 du CGCL.

Mais concrètement, le premier acte de contrôle du conseil de la CL avant l'exécution du budget est symbolisé par l'examen et le contrôle du compte administratif du président de l'exécutif local, par ailleurs, ordonnateur du budget. Contrairement au budget primitif pour lequel l'équilibre est analysé par section, celui du compte administratif est apprécié globalement sur les deux sections et au regard d'un seuil de déficit fixé à $10 \%$ des recettes de la section fonctionnement ${ }^{66}$. Le compte administratif enregistrant les opérations d'exécution

64 Directive $\mathrm{n}^{\circ} 06 / 2009 \mathrm{CM} / \mathrm{UEMOA}$ du 26 juin 2009 relative aux lois de finances.

65 Idem.

66 CISSE (MD) et DIOUF (MP), Manuel de procédure de contrôle de légalité et budgétaire, octobre, 2001, p. 93. 
du budget en vue d'avoir une idée sur la manière dont l'autorité a exécuté le budget, l'assemblée délibérante a un droit de regard sur l'équilibre du compte administratif de l'ordonnateur dans la phase post exécution du budget. En effet, le conseil de la CL vote compte administratif qui est réputé adopté si une majorité ne s'est pas dégagée contre.

Enfin, il y a ce qu'on peut considérer comme le dernier acte de contrôle de l'équilibre des documents budgétaires en cours d'exercice, il s'agit du compte de gestion. En effet, il retrace les flux de recettes et de dépenses effectivement réalisés au cours d'un exercice et établit par le comptable public. Ainsi une simple comparaison des différents flux permet d se rendre compte de l'état d'équilibre de la réalisation du budget.

Dans un souci de transparence pour l'Etat et de proximité pour les CL d'autres acteurs interviennent dans le processus de contrôle budgétaire, il s'agit des citoyens. Lorsque le budget de l'Etat est déficitaire, les citoyens avertis en sont informés par différentes manières. Suivant le marathon budgétaire sanctionné par le vote de la loi de finances qui sera publiée dans le journal officiel, on peut se rendre compte de l'état déficitaire, excédentaire ou équilibré du budget de l'année. Sachant qu'en cas de déficit, le Président de la république est autorisé à recourir à l'emprunt et que l'emprunt présente l'inconvénient de reporter les charges d'aujourd'hui sur les générations futures, le déficit constaté de façon continue dans les budgets qui se succèdent présente, à moins ou long terme, un réel danger pour l'économie nationale. C'est un aspect de contrôle existant. Mais que serait un pouvoir de contrôle sans un pouvoir de sanction? Les citoyens en disposent même si ce pouvoir de sanction ne peut être mis en œuvre que périodiquement c'est-à-dire lors des joutes électorales.

$\mathrm{Au}$ demeurant, il faut noter qu'en pratique les électeurs ne prennent pas en compte cet aspect au moment de faire leur choix électoral. Quoi qu'on puisse dire ce pouvoir de sanction est à la disposition des citoyens.

Au niveau local le contrôle citoyen est plus net. Il s'agit du contrôle exercé par les individus rattachés à la CL par un lien de territorialité. Ici, l'interrogation est de savoir si les individus résidant dans la $\mathrm{CL}$ ont un intérêt à exercer un droit de regard sur un budget voté en déséquilibre? Rappelons que le vote en équilibre réel du budget st une obligation légale qui pèse sur toute CL. Ainsi, l'individu est un acteur du dispositif de contrôle de légalité dont l'aspect budgétaire en est partie intégrante ${ }^{67}$.

Les bases du contrôle citoyen posées, reste à savoir quelles sont les modalités d'exercice du contrôle de l'individu?

On distinguera le contrôle médiat et le contrôle immédiat. Mais avant cela il faut noter les actes préparatoires au contrôle. Parmi ceux-ci, il y a les actes qui tiennent à l'information de l'individu. C'est ce qui ressort de l'article 6 de la section 2 intitulée « Participation citoyenne », « Tout habitant ou contribuable a le droit de demander à ses frais communica-

67 DIAGNE (M), $5^{\text {ème }}$ assise de l'Association ders auditeurs de l'académie international du droit constitutionnel, thème : Le citoyen aujourd'hui. Sous thème traité par M. Diagne : Le citoyen local dans un Etat décentralisé. 
tion, de prendre copie totale ou partielle des procès-verbaux du Conseil régional, du Conseil municipal ou du Conseil rural, des budgets, des comptes et des arrêtés ». Ainsi, le vote d'un budget en déséquilibre est une information accessible à l'individu qui peut alors enclencher une procédure de contrôle direct ou indirect.

S'agissant du contrôle dit médiat il est exercé par le biais généralement d'un organe ou d'une personne. En effet, le citoyen local qui se sent lésé par une délibération du conseil de la CL peut demander au RE de déférer l'acte faisant grief. C'est le déféré provoqué. Le déféré étant l'acte par lequel le RE demande à la Cour suprême d'annuler l'acte d'un élu local.

Ainsi, le CGCL donne au citoyen la possibilité de faire déférer l'acte qui lui parait irrégulier et le budget voté en déséquilibre en fait partie justement. Certes l'initiative revient au citoyen, mais il incombe au RE de saisir le juge. C'est alors une action indirecte de saisine de la Cours suprême par l'entremise du RE. Faut-il le rappeler, le refus du RE de déférer un acte sur demande d'un administré se sentant lésé n'est pas susceptible de recours pour excès de pouvoir. En effet, à la question de savoir si le préfet avait l'obligation de déférer un acte dont la légalité lui semblait enfreinte, le Conseil constitutionnel a, dans une décision du 25 févier 1982, présenté comme une «faculté » l'exercice du déféré. Dans cette même logique le Conseil d'Etat estime que ce refus est insusceptible de recours pour excès de pouvoir dans son arrêt du 25 janvier 1991 « Brasseur ». Ainsi a-t-on pu parler d' « acte de gouvernement au petit pied» ou de pouvoir discrétionnaire et non de compétence liée ${ }^{68}$.

Quant au contrôle dit immédiat, il est exercé directement par le citoyen sans intermédiaire. En premier lieu, nous citerons le recours pour excès de pouvoir qui est un outil de contestation contentieuse pouvant être utilisé par l'individu à raison de son caractère d'ordre public et de son utilité publique ${ }^{69}$. A l'individu qui en est l'auteur, il peut lui être reproché de ne pas avoir intérêt à agir. Apparemment, le lien de territorialité ne suffit pas, il faut aussi être un contribuable local.

Selon le Doyen Bockel, « le contribuable local participe à la couverture des dépenses publiques. Il a donc un intérêt patrimonial à ce que ces dépenses soient régulières. Mais cet intérêt doit être suffisamment individualisé pour que le recours puisse être admis contre une mesure irrégulière ayant des conséquences financières... $\rangle^{70}$

En plus de tous ces moyens de contrôle et d'action donnés aux collectivités locales, il faut mettre en évidence le contrôle latent et menaçant. C'est en effet le contrôle exercé par le biais de la carte électeur qui se présente de plus en plus comme une arme très redoutable face aux actes contraires à la « bonne gouvernance locale » en général, des élus locaux. ${ }^{71}$

69 CE 17 février 1950 « Dame Lamotte». Selon le juge, le REP est un recours objectif et d'ordre public.

70 BOCKEL (A), droit administratif, NEA, Dakar 1978, p. 467.

71 DIAGNE (M), in « Le citoyen dans la décentralisation », op. cit. 
Un autre type de contrôle côtoie et complète le contrôle non juridictionnel, c'est le contrôle juridictionnel.

Il est nécessaire de faire remarquer dès à présent que le législateur sénégalais est revenu sur l'option de spécialisation entamée en 1992. En effet, la Cour Suprême est restaurée et qui est constituée de la Cour de Cassation et du Conseil d'Etat. La nouvelle Cour suprême se voit donc amputée les compétences de la Cour des comptes et du Conseil constitutionnel. Dans la mesure où nos propos portent sur la matière financière, nous distinguerons le contrôle du juge des comptes (paragraphe $1^{\mathrm{er}}$ ) du contrôle exercé par les autres juges (paragraphe 2).

Au Sénégal, c'est la loi 2012-23 du 27 décembre abrogeant et remplaçant celle 99-70 du 17 février 1999 qui régit la Cour des comptes. Cette création est le couronnement d'une série de reformes judiciaires mettant en place de hautes juridictions spécialisées dans leurs tâches ${ }^{72}$. La combinaison des articles 68 et 96 de la constitution du 22 janvier 2001 renseigne des attributions de la Cour des comptes. Les dispositions visées posent que « La Cour des comptes assiste le Président de la république, le Gouvernement et l'Assemblée nationale, dans le contrôle de l'exécution des lois de finances » et « La Cour des comptes juge les comptes des comptables publics. Elle vérifie la régularité des recettes et des dépenses et s'assure du bon emploi des crédits, fonds et valeurs gérés par les services de l'Etat ou par les autres personnes morales de droit public. (...) Elle sanctionne les fautes de gestion commises à l'égard de l'Etat, des collectivités locales et des organismes soumis à son contrôle ».

En plus, il faut signaler le contrôle de la Cour de comptes qui n'est pas juridictionnel. Ce type de contrôle est prévu par la section 3 du chapitre III qui est intitulé «compétence de la cour » dont l'article 43 alinéa 2 dispose que «Ce contrôle englobe tous les aspects de la gestion ${ }^{73}$. C'est donc un contrôle global. Il est ainsi tout à fait loisible à la Cour des comptes de s'appesantir sur l'équilibre réel du budget et sur la sincérité des comptes administratifs et des comptes de gestion. Il peut aussi se pencher sur le contrôle de l'équilibre du budget de l'Etat. En effet, dans sa mission d'assistance prévue à l'article 26 de la loi sur la Cour de comptes, elle peut indiquer aux autorités de l'Etat les voies et moyens pour monter un budget en équilibre. Cela devrait procéder d'un contrôle a priori. Aussi, la Cour des comptes peut « déceler toute irrégularité (...) par rapport aux normes juridiques (...) en vigueur de manière à permettre, dans chaque cas, de prendre les corrections nécessaires, (...) ou de décider des mesures propres à éviter pour l'avenir la répétition de tels actes $\gg^{74}$. En fait, le principe de l'équilibre peut bien faire l'objet d'une investigation approfondie et découler sur la publication d'un rapport particulier.

Tel pourrait être un aspect du contrôle de l'équilibre du budget de l'Etat.

72 Il s'agit de l'éclatement de la Cour Suprême en 1992qui a donné lieu à la mise en place de trois juridictions à savoir le Conseil Constitutionnel, le Conseil d'Etat et la Cour de Cassation.

73 Loi 2013-10 du 28 décembre 2013 portant général des collectivités locales, op. cit.

74 Loi 2013-10 du 28 décembre 2013 portant général des collectivités locales, op. cit. 
Pour ce qui est du budget des CL, le contrôle de l'équilibre réel trouve une bonne assise dans la disposition suivante «Elle (la Cour des comptes) s'assure de la régularité des opérations de recettes et de dépenses des organismes contrôlés et, le cas échéant, réprime les manquements aux règles qui régissent lesdites opérations $\gg{ }^{75}$. Ainsi, l'équilibre réel entre pleinement dans le champ de contrôle de la Cour de comptes sachant qu'il est une des règles principales qui régissent toutes les opérations budgétaires des CL. Etant donné que le comptable de la CL est le receveur principal de la perception du ressort de la CL. En ce sens, il rend compte de sa gestion à la Cour des comptes. Pour ce faire donc, le contrôle de la Cour sera basé sur le compte de gestion qui enregistre la globalité des opérations de l'ordonnateur, président de l'exécutif local. La Cour suspend son contrôle a posteriori de l'exécution des budgets des CL à la production des comptes de gestion. C'est ainsi que par usage obligatoire, cinq mois après la clôture de la gestion les comptables doivent produire toutes les pièces justificatives des opérations effectuées assorties du compte de gestion. Par déduction donc, le compte de gestion peut être irrégulier par le fait de retracer des opérations budgétaires effectuées sur la base d'un budget voté en déséquilibre.

Faut-il préciser que ces comptes atterrissent directement sur la table de la Chambre des Affaires Administratives des Collectivités Locales (CAACL), qui est seule compétente pour juger les comptes de gestions des comptables principaux. Mais à la lecture du rapport public de 2004 de la Cour, il est toujours possible de noter des remontrances à l'encontre des CL qui ne fournissent pas les pièces nécessaires au bon contrôle du budget. La cour parle de l'absence de la reddition des comptes. C'est ainsi que le Conseil Régional de Fatick a été rappelé à l'ordre par la chambre « pour l'absence de documents essentiels que sont les budgets primitifs des gestions 1997, 1999, et 2000 dans l'arrêt n ${ }^{\circ}$ 19/2004 du 24 mars 2004» ${ }^{76}$. Il en est de même dans l'arrêt nº8/2004 du 07 janvier 2004, Communauté rurale de Réfane, où le juge des comptes constate « une absence de pièces générales en plus des pièces justificatives de recettes et des dépenses, les comptes administratifs... ${ }^{77}$. Dans ces arrêts, la Chambre a retenu des griefs d'irrégularités sanctionnant une carence. Et au regard de l'article 29 de la Loi 2013-10 du 28 décembre 2013 portant général des collectivités locales, cette carence est constitutive d'illégalité, car selon cet article, tout comptable public doit rendre compte de sa gestion devant la Cour.

A sein de la Cour des comptes, un autre type de contrôle apparaît plus direct c'est-à-dire immédiat, sans intermédiaire exercé par la Commission de Vérification et de Contrôle de Comptes des Etablissements Publics (CVCCEP) ${ }^{78}$.

«Le Président de la Cour des comptes peut également confier à la Commission de vérification des comptes et de contrôle des entreprises publiques, le soin de s'assurer sur pièces et sur place du bon emploi des crédits, fonds et valeurs gérés par les services de l'Etat, les

Loi 2013-10 du 28 décembre 2013 portant général des collectivités locales, ibidem.

76 Rapport public de la Cour des Comptes de 2004, p. 31.

77 Ibidem.

78 Il s'agit de la troisième formation de la Cour des Comptes. 
collectivités locales et les établissements publics ${ }^{79}$. C'est en ces termes que le législateur confierait à cette commission un pouvoir de contrôle immédiat qui ne fait pas cependant obstacle au contrôle qui peut être effectué de façon médiate.

Le contrôle juridictionnel n'étant pas exclusivement dévolu au juge des comptes, il est nécessaire de voir qu'en est-il pour le juge du Conseil d'Etat dont les attributions sont dévolues à la nouvelle Cour suprême ${ }^{80}$.

En ce qui concerne l'Etat, le contrôle du principe de l'équilibre budgétaire exercé par le juge administratif est presque inopérant ${ }^{81}$. Il convient de faire remarquer dès à présent que les attributions du Conseil d'Etat sont dévolues à la nouvelle Cour suprême qui recueille en même temps les compétences de la Cour de cassation.

On constatera à la suite de la loi organique $n^{\circ} 2008-35$ sur la cour suprême en son article 2 que la CS connaît par voie de recours en cassation contre les décisions de la Cour des comptes $^{82}$. Ainsi, en pratique, le juge administratif n'est directement impliqué dans le contrôle de l'équilibre budgétaire que dans les rares cas où la Cour des comptes statue et qu'à la suite on saisisse la CS en cassation.

De toutes les façons, la fouille de la jurisprudence de la $3^{\text {ème }}$ section créée en 1964, de l'ancienne CS ne révèle aucun cas de recours en cassation portant sur l'équilibre du budget de l'Etat ${ }^{83}$.

Par ailleurs, une nette implication du juge administratif dans le contrôle de l'équilibre budgétaire est aisément remarquée dans l'application du principe de l'équilibre du budget au niveau du droit budgétaire local. Dans ce contrôle de l'équilibre réel du budget des CL, le CE a fait preuve d'une grande perspicacité. Ainsi, en cas de doute sur les recettes, il peut négativement sanctionner la CL.

C'est ainsi que dans l'affaire commune de la Gorges-les Gonesse du 09 juillet 1997, le CE français note : " pendant trois exercices successifs, les recettes communales inscrites à cinq chapitres différents sont restées constantes sans que la commune ne fournisse des explications convaincantes et circonstanciées de nature à faire douter de la réalité des recettes $^{84}$. Le contrôle du CE est allé plus loin. C'est pourquoi dans la note de la décision du 16 mars 2001, Commune de Renne-les-Bain, le juge administratif a été amené à définir les contours de l'équilibre. Depuis lors, est en déséquilibre aux yeux du CE « le budget dans

79 Loi 2013-10 du 28 décembre 2013 portant général des collectivités locales, op. cit.

80 Loi constitutionnelle 2008-34 du 08 aout 2008 portant modification de la constitution (créant la cour suprême).

81 SY Yoro Bocar, président de la $3^{\text {ème }}$ section de la CS du Sénégal, « La jurisprudence de la CS du Sénégal et droit financier », In les CS en Afrique, Tome II, Economica, p. 237-244.

82 Loi organique $\mathrm{n}^{\circ} 2008-35$ du 8 aout 2008 sur la CS.

83 L'arrêt Sokhna contre Etat du Sénégal du CE du 25 septembre 2003 constitue le premier que le CE rend en tant que juge de cassation des arrêts de la Cour des Comptes.

84 CE Section, Commune de Gorges-les Gonesse, RFDA 1998, p. 1048, note Lafargue. 
lequel sont inscrit des subventions incertaines et des emprunts dont la conclusion est très aléatoire compte tenu de l'endettement très élevé de la commune ${ }^{85}$.

Malgré l'enchevêtrement des acteurs du contrôle de l'équilibre budgétaire, vu dans tous ses aspects, il donne l'impression d'un cérémonial c'est-à-dire un contrôle formel. C'est la raison pour laquelle nous nous demandons si ce principe garde toute sa raison d'être en droit sénégalais, si la réponse est négative, adapter la législation en vigueur à la pratique budgétaire surtout au niveau local.

\section{B - La portée du contrôle du principe de l'équilibre budgétaire.}

Ce formalisme du contrôle de l'équilibre doit être cherché tout en conservant la dichotomie des catégories de contrôle. Ainsi, on notera que le caractère formel se manifeste non seulement dans le contrôle juridictionnel, mais aussi dans le contrôle non juridictionnel.

Au sein de la communauté ouest africaine, on se rendra compte que le contrôle juridictionnel de l'équilibre budgétaire n'existe pas à vraiment dire. Là, reviendra la lancinante question de la négation du droit international public par ses détracteurs. Selon eux, l'inexistence de juridictions internationales aptes à prendre des décisions effectives serait la justification de cette négation. Mais on a assisté à la création de la Cour de justice de l'union qui a une compétence large. Ainsi selon Youssouf Any Mahaman, « la Cour de Justice de l'UEMOA est la gardienne du Traité au regard de ses attributions $»^{86}$. C'est donc ce traité, donnant de bases juridiques à l'acte additionnel pris par la Conférence des chefs d'Etats et de Gouvernement sous le numéro $n^{\circ} 04 / 99 / \mathrm{CM} / \mathrm{UEMOA}$, qui prévoit la limitation du déficit budgétaire à $5 \%$ du PIB de chaque Etat membre. Associé au Pacte de convergence, de stabilité, de croissance et de solidarité qui est initié pour lutter contre l'indiscipline budgétaire,

85 CE Section, 16 mars 2001, Commune de Renne-les-Bains, Grands arrêts de la décentralisation, $2^{2 \mathrm{ème} e}$ édition Dalloz, 2001, p. 402. De plus, il existe en fin un autre prétexte de contrôle de l'équilibre du budget des CL par le CE même s'il est indirect. En effet l'article 40 de la loi organique sur la Cour des Comptes dispose que « Tout arrêt définitif rendu par une chambre peut également sur le pourvoi du comptable, des ministres concernés ou du représentant légal de l'organisme dont dépend le comptable, être soumis à cassation pour cause d'incompétence, de vice de forme ou de violation de la loi. Ce pourvoi est formé devant le Conseil d'Etat dans le moi de notification de l'arrêt ». Il existe en fin un autre prétexte de contrôle de l'équilibre du budget des CL par le CE même s'il est indirect. En effet l'article 40 de la loi organique sur la Cour des Comptes dispose que « Tout arrêt définitif rendu par une chambre peut également sur le pourvoi du comptable, des ministres concernés ou du représentant légal de l'organisme dont dépend le comptable, être soumis à cassation pour cause d'incompétence, de vice de forme ou de violation de la loi. Ce pourvoi est formé devant le Conseil d'Etat dans le moi de notification de l'arrêt ». Le CE peut profiter de cette saisine pour « imprimer une orientation à la ligne jurisprudentielle de la Cour des Comptes » selon les termes du docteur Fara Mbodj. En d'autres termes, le CE peut valablement connaître du contentieux relatif à la méconnaissance de la règle de l'équilibre réel du budget local par le biais de la cassation des décisions de la chambre des affaires administratives et des collectivités locales de la Cour des Comptes. Pour ceci voir CE Section, 25 septembre 2003, Sokhna contre Etat du Sénégal.

86 Youssouph Any Mahaman est juge au sein de la Cour de justice de l'UEMOA. 
on s'aperçoit d'une panoplie de textes allant dans le sens de la protection des dispositions relatives au déficit budgétaire des budgets des Etats membres.

Simplement, depuis sa création; la Cour de justice de l'union n'a jamais été saisie de recours tendant à incriminer un Etat dont le budget aurait un déficit excédent la barre des 3 ou 5\% même s'il en existe réellement. Aussi, à supposé que la Cour soit saisie, serait-elle en mesure de prendre des sanctions effectives pouvant ramener l'Etat fautif à la raison et dissuader par là même les autres Etats? En pratique il n'existe pas de cas référentiel, mais dans la logique du droit comparé on pourrait donner l'exemple de la France qui a connu un déficit cyclique pendant longtemps et que ses paires au niveau de l'union européennes n'ont pu que réprimander et rappeler à la raison. Il a fallu attendre l'arrivée du président Sarkozy, qui en a fait une promesse électorale, pour voir enfin la France prendre de réelles décisions politiques tendant au retour de l'équilibre du budget.

Récemment, la Grèce à été désignée « plus mauvais élève de l'union » en raison d'un taux de déficit qui avoisine les 13\% de son Produit Intérieur Brut alors que la barre est fixée à $3 \%$ du PIB. Là aussi on aura assisté à des appels solennels du Conseil des Ministres de l'union pour exhorter l'Etat en question à prendre des mesures idoines pour le retour à la normale.

A ce laxisme dans le contrôle de l'équilibre des budgets des Etats membres de la Communauté, il faut ajouter l'impossibilité pour les personnes physiques de saisir directement la Cour de justice. En effet ils ne peuvent le faire que par le biais de leur Etat, auteur de l'illégalité dont ils font l'allégation. On imagine donc toute la difficulté qu'il y a de pouvoir incriminer un Etat qui va au-delà du plafond légal à savoir 3\% de son PIB. Au demeurant, il faut rappeler le fait que les institutions internationales butent toujours sur les principes d'indépendance, de souveraineté et de non ingérence des Etats membres.

En outre, le droit international public se distingue à la fois de la « courtoisie internationale » et de la « morale internationale $»^{87}$ disais David RUZIE. Il poursuit toujours que « l'interdiction du recours à la force et l'efficacité limitée du pouvoir de sanction des organisations internationales contribuent à l'affaiblissement du droit international $»^{88}$

En définitive, nous remarquerons que sur le plan international le contrôle n'est que formel car ne présentant aucune forme de sanctions effectives, réellement répressives ou dissuasives.

Face à cette situation d'impuissance des juridictions internationales, nous verrons qu'en est-il de celles nationales dans le contrôle de l'équilibre budgétaire. Ici, la principale juridiction est la Cour des comptes. Une lecture croisée du chapitre $1^{\text {er }}$ de la loi 99-70 du 19 février 1999 sur la Cour des comptes ${ }^{89}$ relative aux missions de ladite Cour et du chapitre 3 qui évoque les quatre principales compétences permet de déterminer le champ d'action de

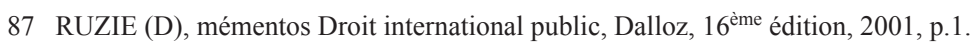

88 Idem.

89 Loi organique 2012-23 du 27 décembre 2012 abrogeant et remplaçant la loi 99-70 du 17 février 1999 sur la Cour des Comptes, op, cit. 
cette juridiction nationale. Un champ d'action réellement existant, mais dans la pratique est ce que ce contrôle est effectif?

Déjà au niveau de l'article 3 de la loi sur la Cour des comptes on stipule parmi ses missions la sauvegarde du patrimoine public et le contrôle de la sincérité des finances publiques $^{90}$. On sait que la Cour a une possibilité de contrôle $a$ priori, en cours d'exercice sur pièce et sur place et a posteriori. Il est important de savoir à quel moment ce contrôle s'avère t-il le plus pertinent et efficace pour le principe de l'équilibre. A priori, on se rendra compte que la Cour serait mise à l'épreuve dans son contrôle par l'autorisation qu'est faite au Président de la république de recourir à l'emprunt, c'est le vote de l'article d'équilibre ${ }^{91}$. Ici la Cour ne pourra pas intervenir car l'équilibre «virtuel » pourvu est légal. En ce qui concerne le contrôle en cours d'exercice, il faut rappeler la Cour est juge des comptes des comptables publics. Il faut se demander si les comptes d'un comptable tenus en application d'une loi de finances englobant un budget déficitaire, seraient entaché d'irrégularités aux yeux de la Cour? La réponse serait non car elle n'a compétence ici qu'à contrôler l'adéquation de résultats par rapport aux autorisations données par Parlement. Enfin, concernant le contrôle a posteriori la Cour détient l'ultime occasion de faire des remarques sur l'ensemble du processus budgétaire de l'adoption au contrôle en passant par l'exécution. En effet, le rapport public annuel est un moment solennel que la Cour peut saisir pour revenir sur la situation budgétaire et économique du pays. Le cas échéant, il peut émettre des avis sur la situation de l'équilibre budgétaire et économique de l'Etat. Ainsi on peut évoquer les remarques faites par la Cour des comptes française dans son rapport de 2009. En marge de son avis sur la crise économique ayant secoué la monde en 2008, la Cour a préconisé une baisse du déficit public ${ }^{92}$. Là aussi, on se rend compte que le contrôle du principe de l'équilibre budgétaire exercé par la Cour des comptes est qu'un rituel pour ne pas dire inopérant.

Appréciant maintenant le contrôle juridictionnel du budget des collectivités locales, nous analyserons de près celui exercé par la Cour des comptes par le biais de sa chambre des affaires administratives des collectivités locales (CAACL). Aux termes des dispositions de la loi numéro 2012-23 du 27 décembre 2012 sur la cour des comptes « cinq mois après la clôture de chaque gestion, les comptables publics sont tenus de présenter leurs comptes de gestion accompagnés de toutes les pièces justificatives par l'intermédiaires de leur supérieur hiérarchique qui s'assure que les comptes sont en état $»^{93}$.

L'examen du rapport de la Cour des comptes permet de se rendre compte de l'insuffisance du personnel de contrôle au sein de cette cours d'où le doute sur l'efficacité de ce contrôle. En effet, la juridiction financière compte globalement 40 agents de contrôle pour

90 Idem.

91 DC numéro DC 79-111 du 1979 du 30 décembre 1979, Revue des Arrêts du Conseil Constitutionnel français.

92 Rapport public annuel de la Cour des comptes française, www.ccomptes.fr.

93 Loi organique 2012-23 du 27 décembre 2012 abrogeant et remplaçant la loi 99-70 du 17 février 1999 sur la Cour des Comptes, op, cit.. 
$441 \mathrm{CL}$ directement soumise au contrôle de la $\mathrm{CAACL}^{94}$. Soit quarante (40) CL à contrôler pour chaque agent. Ce qui est matériellement impossible si on doit effectuer des contrôle cours de gestion et vue la technicité et la pléthore de documents à exploiter pour un contrôle sérieux. Ce manque de ressources humaines est de nature à limiter la portée du contrôle exercé par la Cour des comptes sur les CL. L'hypothèse de la dégénérescence de la mission de contrôle de la Cour des comptes est envisageable ${ }^{95}$. En effet, il est à craindre que la Cour des comptes serve de chambre d'enregistrement des comptes des CL. Tout ceci constitue une véritable limite à l'effectivité du contrôle juridictionnel de la Cour des comptes ${ }^{96}$. Il s'y ajoute que dans plusieurs CL, les comptes produits ne sont pas souvent en état d'être jugés.

Les défaillances existent et persistent. En effet, dans son rapport de 2004, la Cour des comptes rappelle en en son chapitre 4 consacré à l'activité de la CAACL que « au 31 décembre 2004, la CAACL n'a pas enregistré une évolution notable dans son activé juridictionnelle par rapport à 2003, en raison, principalement, de la persistance des contraintes liées à la reddition des comptes des CL ». En effet, les comptes de gestion de 164 CL n'ont pas été produits pour les années 1997, 1998, 1999, 2000, 2001, 2002 et 2003. Et même les CL ayant produit effectivement leurs comptes, aucunes d'entre elles n'a présenté des comptes en état d'être jugés. Devant cette situation, la CAACL a pris une série de trois types d'arrêts :

- Arrêts ordonnant le retour des comptes;

- Arrêts ordonnant la poursuite de l'instruction et;

- Arrêts ordonnant l'instruction pour le retour d'une partie des comptes et la poursuite de l'instruction pour l'autre partie ${ }^{97}$.

Il manque à une large échelle, l'existence de comptes administratifs régulièrement tenus. Et si de tels documents existent, ils sont en général la pâle copie des comptes de gestion. Il convient même de faire remarquer l'inexistence de comptes administratifs dans certaines $\mathrm{CL}^{98}$.

Le rituel se manifeste également par le recours en 1996 d'un apurement sur chiffre emportant un jugement des comptes sur 20 ans $^{99}$.

94 Rapport public général de la Cour des Comptes de 2003.

95 Rapport public 2004 de la Cour des comptes, p. 11.

96 MBODJ (F), Revue « droit sénégalais » $\mathrm{n}^{\circ} 7$ novembre 2008, page 317 et suivant, Presse de 1’Université des Sciences Sociales de Toulouse, op.cit.

97 Face à cette situation qui perdure, la CAACL a déclenché en avril 2002, sur l'initiative du commissaire du droit, en sa qualité représentant du ministère public, une procédure d'amende pour retard contre 55 comptables public de CL dont les communes de Kolda, Kaolack, Gossas, Foundiougne, Nioro du Rip, Guinguinéo, Dahara, et Kaone et la région de Kolda. Voir Rapport public 2006.

98 Rapport de la Cour des comptes année 2001.

99 Rapport de la Cour des comptes année 2001, op.cit. p 27. 
Toutes ces considérations font penser que le contrôle juridictionnel prend la forme d'un rituel, d'un cérémonial au détriment de sa fonction originelle. L'absence de pièces essentielles, le dépôt tardif des comptes, l'insuffisance du personnel de contrôle n'exclus certes pas le contrôle, mais il est à craindre que de telles considérations arrivent à vider le contrôle de son sens. La conséquence en est que les contrôles peuvent se répéter en ne conservant qu'un cachet psychologique et symbolique signe de rituel. Ce rituel relègue le droit à une place qui ne doit pas être la sienne. A terme, la couverture juridictionnelle de la Cour des comptes n'est pas encore satisfaisante ${ }^{100}$.

Si le contrôle de l'équilibre budgétaire éprouve des difficultés dans le cadre même des juridictions, il est très probable qu'il connaisse ce même problème en ce qui le concerne le contrôle non juridictionnel. Ce que nous verrons dans l'argumentaire suivant.

On étudiera tour à tour le contrôle de l'équilibre du budget de l'Etat et du budget des CL. Pour ce qui est du budget de l'Etat, rappelons que ce contrôle est exercé par le Parlement, et des organes comme l'Inspection Générale d'Etat (IGE) et le Contrôle Financier (CF), mais aussi dans une moindre mesure, ce bel oxymoron, le contrôle non juridictionnel de la juridiction des comptes.

S'agissant du contrôle exercé par le Parlement sur l'application du principe de l'équilibre dans le budget de l'Etat, on notera une intervention a priori et une intervention a posteriori de celui-ci. Dans le sens de son intervention avant l'exécution du budget on se rendre compte qu'au lieu de contrôler véritablement l'exécutif, le Parlement continue de jouer son rôle de chambre d'enregistrement et, plus même, joue le rôle de complice dans le « contournement organisé » du principe de l'équilibre budgétaire. En effet, c'est le Parement lui-même qui autorise le Gouvernement à recourir à l'emprunt à la hauteur du déficit considéré. C'est le vote du Chapitre d'équilibre illustré par un arrêt du Conseil Constitutionnel qui pose que « qu'en vertu de l'article 40 de l'ordonnance du 2 janvier 1959 portant loi organique relative aux lois de finances, la seconde partie de la loi de finances de l'année ne peut être mise en discussion devant une assemblée avant le vote de la première partie $»^{101}$.

C'est ce consentement explicite du Parlement sur la nature du budget qui dénude de tout sens le contrôle, en cours d'exécution, du principe de l'équilibre. En effet, il serait incohérent de vouloir poser des questions soit écrites ou orales sur la situation de l'équilibre du budget, alors que c'est ce même organe qui avait autorisé le recours à l'emprunt afin de couvrir le déficit.

Pour ce qui est du contrôle après l'exécution du budget, le Parlement ne détient que cet instant privilégié et solennel qu'est le vote de la loi de règlement. Cette loi est donc plus une loi de bilan qu'une loi d'appréciation de l'équilibre du budget de l'Etat. Pour ce qui est

100 MBODJ, (F), op.cit.

101 DC 79-111 du 1979 du 24 décembre 1979 « Considérant qu'en vertu de l'article 40 de l'ordonnance du 2 janvier 1959 portant loi organique relative aux lois de finances, " la seconde partie de la loi de finances de l'année ne peut être mise en discussion devant une assemblée avant le vote de la première partie $»$. 
du budget des CL nous mettrons l'accent sur le contrôle exercé par le représentant de l'Etat sans pour autant occulter le contrôle rapproché qui est celui exercé par l'organe délibérant de la CL elle même.

Le RE joue un rôle très important dans le dispositif de contrôle des CL. En effet, c'est au RE d'exercer un contrôle de proximité, c'est-à-dire un contrôle de suivi des actes des CL. Le vote en équilibre réel du budget des CL est un acte qui s'inscrit dans le processus budgétaire. A ce titre, il est soumis à l'approbation préalable du RE en vue de donner au budget un caractère exécutoire ${ }^{102}$. Comment se manifeste alors le rituel dans l'exercice du contrôle du RE?

Le rituel dans le contrôle dévoile des pratiques en marge du contrôle ${ }^{103}$. C'est ainsi que le contrôle du RE sur l'équilibre réel du budget local reste un contrôle de l'équilibre virtuel porté sur l'artifice comptable symbolisé par le mouvement financier.

Le recours systématique et généralisé au mouvement financier dénature l'équilibre réel du budget local. Le contrôle du RE sur le budget local ne tient pas compte de la configuration comptable réelle du budget. C'est alors un contrôle rituel fondé sur le fait que le contrôle est formellement aménagé, il convient alors de satisfaire cette exigence légale sans donner au contrôle sa « dimension légale».

Il s'y ajoute l'inscription de recettes douteuses de subventions incertaines ou de recettes aléatoires. Le rituel est également observable par l'inscription constante de certaines dépenses et de certaines recettes sur plusieurs exercices répétés.

Touts ces pratiques informelles réduisent le contrôle du RE à un contrôle rituel.

Sachons que le contrôle non juridictionnel du budget de la CL est aussi une affaire de l'organe délibérant. Il est vrai que c'est le même organe qui autorise par vote le budget de la $\mathrm{CL}$, c'est la raison pour laquelle il est appelé à contrôler en premier lieu l'équilibre du budget de la CL. Mais la proximité entre l'organe de contrôle et l'organe contrôlé, voire même la confusion dénote un contrôle pas aisé. Aussi, le fait que ce budget soit malgré cela, de temps à autre, voté en déséquilibre nous fait assimiler ce contrôle rituel ${ }^{104}$.

Enfin, toutes ces considérations bousculent l'obligation légale de voter le budget des $\mathrm{CL}$ en équilibre réel. Ainsi, est-il pertinent de se demander s'il faut continuer à l'ériger en principe obligatoire pour les CL dès lors que tous les indicateurs d'un déséquilibre certains sont visibles? De ce fait, le budget local serait dans la trajectoire d'un « équilibre psychologique » à l'image du budget de l'Etat.

Donc, ne faut-il pas réfléchir sur la nécessaire coïncidence entre textes régissant le budget local et ce qui se fait en réalité? D'où cette dernière approche qui se présente sous forme de perspective sur la législation financière locale. Un véritable plaidoyer!

102 Code général des collectivités locales du Sénégal, on note la subsistance de la tutelle financière sur les CL d'avant les lois de la décentralisation dans son article 243.

103 MBODJ (F), Revue « droit sénégalais " ${ }^{\circ} 7$ novembre 2008, page 317 et suivant, Presse de l'Université des Sciences Sociales de Toulouse, op.cit.

104 Voir BELLE (J), Revue française des finances publiques, LGDJ nº 85, février 2004, article intitulé « l'oxymore » sur la manière dont il conçoit les types de contrôle internes et externes. 
Pour une adéquation entre législation budgétaire locale et réalité budgétaire locale.

A partir du moment où les textes posés ne sont pas appliqués à la lettre et que les acteurs parviennent toujours à les contourner soit pour des difficultés de gestion, soit par nécessité, il est important de s'interroger sur l'actualité de cette norme. Ne devant pas se satisfaire de ce constat et permette, à l'avenir, que tous ces agissements (mouvement financier, budget excédentaire) entrent dans le champ de la légalité, nous réfléchirons d'abord sur les enjeux de la réorientation envisagée avant de montrer les voies préconisées pour cette même adaptation.

De prime à bord, disons qu'il y a des intérêts considérables à faire en sorte que les lois de la décentralisation telles que libellées après plusieurs années de réflexions recoupent toute la réalité dans leur application au niveau local. L'intérêt est d'abord théorique. Après plus d'une décennie de d'application des lois de la décentralisation, il est intéressant de dresser le bilan. Car, la perfection d'un texte n'est appréciable que s'il est mis application. De là, ressort toutes les failles et inconvenances qui peuvent à l'avenir être rectifiées ${ }^{105}$. C'est ainsi que la problématique de l'effectivité d'une législation applicable et appliquée est d'actualité au Sénégal. On assisterait à la diminution des droits du RE qui verra les pratiques informelles « jadis considérées comme un décalage par rapport à la légalité ».

Dans la pratique, l'enjeu est beaucoup plus manifeste pour les CL. En effet, cela permettrait à celles-ci un allégement de l'obligation légale lourde et quasi irréalisable. C'est le cas de l'équilibre réel des budgets des CL qui est dans nos textes magnifié par l'article 255 du CGCL ${ }^{106}$. Aussi, cela constituerait une réponse face à la vieille demande des élus locaux qui consiste à vouloir plus de responsabilités surtout dans la gestion des finances locales. Une telle adéquation laisserait espérer l'amélioration des services sociaux de base que sont en droit d'attendre les citoyens locaux et à terme le sénégalais lambda. En effet, certaines $\mathrm{CL}$, ayant la capacité requise pourront contracter des dettes ou recourir à l'emprunt pour la réalisation de certains travaux et d'équipements, base d'un développement durable. Toujours, pourrait-on les permettre de pouvoir de ponctionner sur la section fonctionnement au profit de la section investissement et vice versa, en fonction des priorités et des nécessités du moment.

Pour l'Etat, l'enjeu serait déjà de donner du tonus au $\mathrm{PIB}^{107}$ et même à la longue diminuer les subventions allouées aux CL. Cette perspective a certes des risques d'effets pervers notamment le développement non harmonieux des CL. Les CL les plus en santé financière seraient plus à même de pouvoir recourir aux voies de financement indirect tel que l'emprunt.

105 Voir Rapport général des assises sur la décentralisation au Sénégal les 27,28 et 29 novembre 2007.

106 Loi n²012-10 du 28 décembre 1012 portant CGCL du Sénégal.

107 Le produit intérieur brut (PIB) est un indicateur économique très utilisé, qui mesure le niveau de production d'un pays. Il est défini comme la valeur totale de la production interne de biens et services dans un pays donné au cours d'une année donnée par les agents résidant à l'intérieur du territoire national. 
Cependant, il faut corrélativement mettre en place un système de péréquation efficace et efficient. Un autre risque est celui de la formation des autorités locales affin d'affiner leurs capacités. Car comme le dit M. Etienne TURPIN « former une personne (permet de) faire évoluer ses capacités cognitives, ses aptitudes compétencielles, ses attitudes et ses comportements $»^{108}$. Ceci est, de nos jours d'une nécessité très actuelle face à la demande de plus en plus pressante de pouvoirs accrus par les élus locaux. Mais, à la question de savoir quelles sont les voies de cette réorientation, une réponse se trouvera dans l'argumentaire suivant.

Si l'on est convaincu des enjeux de la nécessaire adéquation entre législation financière locale et pratique budgétaire, il nous faut proposer les canevas pertinents et idoines pour atteindre cet objectif. C'est dans ce sens que nous ferons observer d'abord le scénario politique. Ici, on se rencontre fort heureusement de la coïncidence, pour le plus claire du temps, entre élus locaux et personnalités politiques étatiques. En effet, il est très fréquent de rencontrer au Sénégal des «ministre-maire » ou des " députés-maires ». Ainsi, l'exercice simultané de ces fonctions permet dans un premier temps de pouvoir se rendre compte des obstacles légales confrontés en application des textes destinés aux CL. Dans un second temps, il est attendu de cette autorité locale, par sa proximité avec l'appareil législative, d'introduire une « proposition de loi » allant dans le sens d'assouplir ou de rationaliser les textes appliqués aux CL.

Sachant que toute réforme nécessite une étude sociologique, il faut évidemment tâter le pouls de la population en procédant à des enquêtes ou sondages. Car il y a des chances que les citoyens imbus de la situation et des enjeux financières des collectivités locales, soient hostiles à une modification de la législation tendant à assouplir la règle de l'équilibre. Ensuite, il est important de s'enquérir de l'expérience des autorités administratives dépositaires du pouvoir de contrôle sur le CL.

Techniquement cependant, il faut procéder à des études auprès des directions spécialisées en la matière comme la direction des CL ou la direction générale de l'administration territoriale. Aussi, le Parlement a en son sein des commissions techniques qui peuvent en connaissance de cause relever les avantages et les inconvénients de la proposition ou du projet qui lui est soumis. Faisant allusion aux arguments militant pour l'acceptation d'une telle réforme, il faut rappeler le besoin de coïncider les pratiques informelles en marge de la loi, et une nouvelle législation prenant en compte ces écarts de conduite parfois nécessaires ${ }^{109}$. De plus il faut que la loi réponde à sa définition d'en temps, c'est-à-dire « être le reflet de la société ». En ce sens, la loi doit aussi être le reflet de la réalité au sein des CL et tenir compte de l'évolution socio politique et économique.

Le dernier canevas et plus pragmatique s'avère être celui juridique. Ici, le maître mot est « légiférer ». Dans un autre registre, c'est un beau prétexte pour remodeler l'articulation

108 Voir Rapport général des assises sur la décentralisation au Sénégal les 27,28 et 29 novembre 2007, p.29.

109 Les mouvements financiers, et l'excédent budgétaire. 
organique et matérielle la Cour Suprême et la Cour des Comptes dans l'esprit de la réforme de 1992 qui a jeté les bases de la spécialisation juridictionnelle. En effet nous plaidons pour une réunification du contentieux budgétaire et financier autour de la Cour des comptes de connaitre en dernier ressort de la cassation des arrêts définitifs rendus par ses formation et que les arrêts rendus par celui-ci ne soient pas susceptibles de cassation par le Conseil d'Etat, aujourd'hui la Cour Suprême. Il faut cependant que l'Etat affiche une volonté politique bien réelle. 